\title{
Extended 2-tuple linguistic hybrid aggregation operators and their application to multi-attribute group decision making
}

\author{
Fanyong Meng* \\ School of Management, Qingdao Technological University, \\ Qingdao, 266520, Shandong, China \\ Jie Tang \\ School of Management, Qingdao Technological University, \\ Qingdao, 266520, Shandong, China
}

Received 6 August 2013

Accepted 27 November 2013

\begin{abstract}
The aim of this paper is to develop some new 2-tuple linguistic hybrid aggregation operators, which are called the extended 2-tuple linguistic hybrid arithmetical weighted (ET-LHAW) operator, the extended 2-tuple linguistic hybrid geometric mean (ET-LHGM) operator, the induced ET-LHAW (IET-LHAW) operator and the induced ET-LHGM (IET-LHGM) operator. These operators do not only consider the importance of the elements but also reflect the importance of their ordered positions. Meantime, some desirable properties are studied, such as idempotency, boundary, etc. When the information about linguistic weight vectors is partly known, the models for the optimal linguistic weight vectors on an expert set, on an attribute set and on their ordered sets are established, respectively. Moreover, an approach to multi-attribute group decision making under linguistic environment is developed. Finally, a numerical example is offered to verify the developed method and to demonstrate its practicality and feasibility.
\end{abstract}

Keywords: multi-attribute group decision making; uncertain linguistic value; aggregation operator; TOPSIS method

\section{Introduction}

In the real world there are many situations in which problems are too complex or too ill-defined to be amenable for description in conventional quantitative expressions. The use of numerical based modelling to represent such problems is not always adequate. Often the experts that take part in this type of problems use linguistic descriptors to express their assessments regarding the uncertain knowledge they have about the problem [1-11]. A number of studies have recently focused on using the linguistic variables to model the problems and have produced successful results in different fields [12-21].

In the process of multi-attribute decision making, the linguistic decision information needs to be aggregated by means of some proper approaches so as to rank the given decision alternatives and then to select the most desirable one(s). Herrera and Martínez [5] developed the 2-tuple linguistic arithmetic mean (TLAM) operator, the 2-tuple linguistic weighted averaging (TLWA) operator and the 2-tuple linguistic ordered weighted averaging (TLOWA) operator. Jiang and Fan [22] defined the 2-tuple linguistic weighted geometric (TLWG) operator and the 2-tuple linguistic ordered weighted geometric (TLOWG) operator. Zhang and Fan [23] proposed the extended 2-tuple linguistic ordered weighted averaging (ET-LOWA) operator. Wei et al. [24] presented the 2-tuple linguistic hybrid weighted averaging (T-LHWA) operator. Xu and Huang [25] introduced the 2-tuple linguistic weighted geometric averaging (TLWGA) operator, the 2-tuple linguistic ordered weighted geometric averaging (TLOWGA) operator, and the 2-tuple linguistic hybrid geometric averaging (TLHGA) operator. Wei [26] defined the extended 2-tuple linguistic weighted geometric (ET-LWG) operator and the extended 2-tuple linguistic ordered weighted geometric (ET-LOWG) operator. $\mathrm{Xu}$ and Wang [27] gave the 2-tuple linguistic power average (2TLPA) operator and the 2-tuple linguistic power

*Corresponding author. Tel.: +86-18254298903; Fax: +86-0532-86875851.

E-mail address: mengfanyongtjie@163.com. 
ordered weighted average (2TLPOWA) operator. Meanwhile, Wei [28] developed the generalized 2-tuple weighted average (G-2TWA) operator, the generalized 2-tuple linguistic ordered weighted average (G-2TLOWA) operator, and the induced generalized 2-tuple linguistic ordered weighted average (IG-2TLOWA) operator.

As $\mathrm{Xu}$ and $\mathrm{Da}$ [29] have noted, the ordered weighted operator only considers the importance of the ordered positions, while the weighted operator only gives the importance of elements. In order to both consider the importance of the elements and that of their ordered positions, $\mathrm{Xu}$ and $\mathrm{Da}[30,31]$ defined the hybrid weighted averaging (HWA) operator and the hybrid weighted geometric mean (HWGM) operator. After the pioneer works of $\mathrm{Xu}$ and $\mathrm{Da}$ [30, 31], many hybrid aggregation operators are presented [24, 25, 32-34]. However, all above mentioned hybrid aggregation operators do not satisfy boundary or idempotency, which are desirable properties for aggregating a finite collection of arguments.

If the weight vector is exactly known, then we can use some aggregation operator to get the best choice(s). Sometimes, however, we are only able to provide uncertain information about the weights because of time pressure, lack of knowledge, or data, and their limited expertise related to the problem domain. In these cases, it would be necessary to develop a model to obtain such weight information.

In order to eliminate the issue in the existing hybrid operators, this study develops some new 2-tuple linguistic hybrid aggregation operators called the extended 2-tuple linguistic hybrid arithmetical weighted (ET-LHAW) operator, the induced extended 2-tuple linguistic hybrid arithmetical weighted (IET-LHAW) operator, the extended 2-tuple linguistic hybrid geometric mean (ET-LHGM) operator, and the induced extended 2-tuple linguistic hybrid geometric mean (IET-LHGM) operator. These operators do not only consider the importance of the elements but also reflect the importance of their ordered positions. Furthermore, based on distance deviation method, TOPSIS method and mean deviation method, the models for the optimal linguistic weight vectors are built. Then, an approach to multi-attribute group decision making under linguistic environment is developed.

The remainder of this paper is set out as follows: In Sect. 2, we introduce some basic concepts related to 2-tuple linguistic arguments and some hybrid aggregation operators. In Sect. 3, we define four new 2-tuple linguistic hybrid aggregation operators called the ET-LHAW, ET-LHGM, IET-LHAW and IET-LHGM operators. Meanwhile, some desirable properties are studied, such as commutativity, monotonicity, idempotency, and boundary. In Sect. 4, based on distance deviation method, TOPSIS method and mean deviation method, the models for the optimal linguistic weight vectors on the expert set, on the attribute set and on their ordered sets are established, respectively. In Sect. 5, an approach to multi-attribute group decision making under linguistic environment is developed. In Sect. 6, an illustrative example is presented to verify the developed method. In Sect. 7, the conclusions are made.

\section{Preliminaries}

Let $S=\left\{s_{i} \mid i=1,2, \ldots, t\right\}$ be a linguistic term set with odd cardinality. Any label, $s_{i}$ represents a possible value for a linguistic variable, and it should satisfy the following characteristics [4]:

(1) The set is ordered: $s_{i}>s_{j}$, if $i>j$;

(2) Max operator: $\max \left(s_{i}, s_{j}\right)=s_{i}$, if $s_{i} \geq s_{j}$;

(3) Min operator: $\min \left(s_{i}, s_{j}\right)=s_{i}$, if $s_{i} \leq s_{j}$;

(4) A negation operator: $\operatorname{neg}\left(s_{i}\right)=s_{j}$ such that $j=$ $t-i$.

For example, $S$ can be defined as

$S=\left\{s_{1}\right.$ : extremely poor, $s_{2}$ : very poor, $s_{3}$ : poor, $s_{4}$ : fair, $s_{5}$ : good, $s_{6}$ : very good, $s_{7}$ : extremely $\operatorname{good}\}$.

Herrera and Martínez [5] developed the 2-tuple fuzzy linguistic representation model based on the concept of symbolic translation. It is used for representing the linguistic assessment information by means of a 2-tuple $\left(s_{i}, \alpha_{i}\right)$, where $s_{i}$ is a linguistic label from predefined linguistic term set $S$ and $\alpha_{i}$ is the value of symbolic translation with $\alpha_{i} \in[-0.5,0.5)$.

Definition 2.1 [5] Let $\beta$ be the result of an aggregation of the indices of a set of labels assessed in a linguistic term set $S$, i.e., the result of a symbolic aggregation operation, $\beta \in[1, \mathrm{t}]$, being $t$ the cardinality of $S$. Let $i=\operatorname{round}(\beta)$ and $\alpha=\beta-i$ be two values, such that, $i \in[1, \mathrm{t}]$ and $\alpha \in[-0.5,0.5)$, then $a$ is called a symbolic translation.

Definition 2.2 [5] Let $S=\left\{s_{1}, s_{2}, \ldots, s_{t}\right\}$ be a linguistic term set, and $\beta \in[1, \mathrm{t}]$ be a number value representing the aggregation result of linguistic symbolic, then the 2-tuple that expresses the equivalent information to $\beta$ is obtained with the following function $\Delta$ : 


$$
\begin{aligned}
& \Delta:[1, \mathrm{t}] \rightarrow S \times[-0.5,0.5), \\
& \Delta(\beta)=\left(s_{i}, \alpha_{i}\right), \text { with }\left\{\begin{array}{l}
s_{i}, i=\operatorname{round}(\beta) \\
\alpha_{i}=\beta-i, \alpha_{i} \in[-0.5,0.5)
\end{array},\right.
\end{aligned}
$$

where $\operatorname{round}(\cdot)$ is the usual round operation, $s_{i}$ has the closest index label to $\beta$ and $\alpha_{i}$ is the value of the symbolic translation.

Definition 2.3 [5] Let $S=\left\{s_{1}, s_{2}, \ldots, s_{t}\right\}$ be a linguistic term set, and $\left(s_{i}, \alpha_{i}\right)$ be a 2 -tuple. There is always a function $\Delta^{-1}$ :

$$
\begin{aligned}
& \Delta^{-1}: S \times[-0.5,0.5) \rightarrow[1, \mathrm{t}], \\
& \Delta^{-1}\left(s_{i}, \alpha_{i}\right)=i+\alpha_{i}=\beta .
\end{aligned}
$$

From Definitions 2.1 and 2.2, we can conclude that the conversion of a linguistic term into a linguistic 2-tuple consists of adding a value 0 as symbolic translation:

$$
\Delta\left(s_{i}\right)=\left(s_{i}, 0\right) .
$$

Let $\left(s_{k}, \alpha_{1}\right)$ and $\left(s_{l}, \alpha_{2}\right)$ be any two 2-tuples, Herrera and Martínez [5] gave the following relationship between them.

(1) If $k<l$ then $\left(s_{k}, \alpha_{1}\right)$ is smaller than $\left(s_{l}, \alpha_{2}\right)$, denoted $\left(s_{k}, \alpha_{1}\right)<\left(s_{l}, \alpha_{2}\right)$.

(2) If $k=l$ then,

(a) if $\alpha_{1}=\alpha_{2}$, then $\left(s_{k}, \alpha_{1}\right)$ and $\left(s_{l}, \alpha_{2}\right)$ represent the same information, denoted $\left(s_{k}, \alpha_{1}\right)=\left(s_{l}, \alpha_{2}\right)$,

(b) if $\alpha_{1}<\alpha_{2}$ then $\left(s_{k}, \alpha_{1}\right)$ is smaller than $\left(s_{l}, \alpha_{2}\right)$, denoted $\left(s_{k}, \alpha_{1}\right)<\left(s_{l}, \alpha_{2}\right)$,

(c) if $\alpha_{1}>\alpha_{2}$ then $\left(s_{k}, \alpha_{1}\right)$ is bigger than $\left(s_{l}, \alpha_{2}\right)$, denoted $\left(s_{k}, \alpha_{1}\right)>\left(s_{l}, \alpha_{2}\right)$.

$\mathrm{Xu}$ and $\mathrm{Da}$ [29] found the ordered weighted operator only considers the importance of the ordered positions, but does not give the importance of their own. Based on the OWA operator [35] and the weighted arithmetic averaging (WAA) operator [36], $\mathrm{Xu}$ and $\mathrm{Da}$ [30] developed the hybrid weighted averaging (HWA) operator, which is defined as follows:

Definition 2.4 [30] A HWA operator of dimension $n$ is a mapping HWA: $\mathrm{R}^{n} \rightarrow \mathrm{R}$, which has an associated weight vector $w=\left(w_{1}, w_{2}, \ldots, w_{n}\right)^{T}$ on $N=\{1$, $2, \ldots, n\}$ such that $w_{j} \in[0,1], \sum_{j=1}^{n} w_{j}=1$, denoted by

$$
\operatorname{HWA}_{w, \omega}\left(a_{1}, a_{2}, \ldots, a_{n}\right)=\sum_{j=1}^{n} w_{j} b_{j},
$$

where $b_{j}$ is the $j$ th largest value of the weighted argument $n \omega_{j} a_{j}(j=1,2, \ldots, n), \omega=\left(\omega_{1}, \omega_{2}, \ldots, \omega_{n}\right)^{T}$ is the weight vector of $a_{i}(i=1,2, \ldots, n)$ with $\omega_{i}>0$, $\sum_{i=1}^{n} \omega_{i}=1$, and $n$ is the balancing coefficient.

Based on the geometric mean (GM) operator and the OWG operator [37], $\mathrm{Xu}$ and $\mathrm{Da}$ [31] further developed the hybrid weighted geometric mean (HWGM) operator to aggregate the arguments in a similar way to the HWA operator as follows:

Definition 2.5 [31] A HWGM operator of dimension $n$ is a mapping HWGM: $\mathrm{R}^{n} \rightarrow \mathrm{R}$, which has an associated weight vector $w=\left(w_{1}, w_{2}, \ldots, w_{n}\right)^{T}$ such that

$$
\begin{gathered}
w_{j} \in[0,1], \sum_{j=1}^{n} w_{j}=1, \text { denoted by } \\
\operatorname{HWGM}_{w, \omega}\left(a_{1}, a_{2}, \ldots, a_{n}\right)=\prod_{j=1}^{n} b_{j}^{w_{j}},
\end{gathered}
$$

where $b_{j}$ is the $j$ th largest value of the weighted arguments $a_{j}^{n \omega_{j}}(j=1,2, \ldots, n), \omega=\left(\omega_{1}, \omega_{2}, \ldots, \omega_{n}\right)^{T}$ is the weight vector of $a_{i}(i=1,2, \ldots, n)$ with $\omega_{i}>0$, $\sum_{i=1}^{n} \omega_{i}=1$, and $n$ is the balancing coefficient.

In the real world, there are many situations in which problems must deal with vague and imprecise information that usually involves uncertainty in their definition frameworks. It is hard to provide numerical precise information of the weights of elements and their ordered positions when the knowledge is vague. Often the experts that take part in this type of problems use linguistic descriptors to express their assessments regarding the uncertain knowledge they have about the problem.

Later, $\mathrm{Xu}$ [32] defined the linguistic hybrid geometric averaging (LHGA) operator; $\mathrm{Xu}$ and Huang [25] proposed the 2-tuple hybrid geometric averaging (THGA) operator; Wei et al. [24] presented the 2-tuple hybrid weighted averaging (T-HWA) operator; $\mathrm{Xu}$ [33] introduced the uncertain linguistic hybrid aggregation (ULHA) operator, whilst Wei [34] defined the uncertain linguistic hybrid geometric mean (ULHGM) operator. But all these operators can only be used in the setting of the weights of elements and their ordered positions both taking the form of real numbers. Further, all above mentioned hybrid aggregation operators do not satisfy boundary or idempotency, which are desirable properties for aggregating a finite collection of arguments. For example, let $S=\left\{s_{i} \mid i=1,2,3,4,5\right\}$ and $X=\left\{s_{2}, s_{3}, s_{4}\right.$, $\left.s_{5}\right\}$. Assume that $w=\{0.4,0.3,0.2,0.1\}$ and $\omega=\{0.1$, $0.2,0.3,0.4\}$. By the HWA operator, it has $\mathrm{HWA}_{w, \omega}$ $=\left(s_{2}, s_{3}, s_{4}, s_{5}\right)=s_{5.2}>s_{5}$; by the HWGM operator, it 
has $\mathrm{HWGM}_{w, \omega}=\left(s_{2}, s_{3}, s_{4}, s_{5}\right)=s_{5.6}>s_{5}$. Furthermore, by the HWA operator, it has $\operatorname{HWA}_{w, \omega}=\left(s_{2}, s_{2}, s_{2}, s_{2}\right)$ $=s_{2.4} \neq s_{2}$; by the HWGM operator, it has $\mathrm{HWGM}_{w, \omega}$ $=\left(s_{2}, s_{2}, s_{2}, s_{2}\right)=s_{2.3} \neq s_{2}$.

\section{New extended 2-tuple linguistic hybrid aggregation operators}

In this section, we define four new extended 2-tuple linguistic hybrid aggregation operators for the group decision-making problems, in which the weights of experts, attributes, their ordered positions as well as the attribute preference values all take the form of linguistic information.

Based on the extended 2-tuple weighted average (ET-WA) operator [5] and the extended 2-tuple ordered weighted averaging (ET-OWA) operator [23], we define the following extended 2-tuple linguistic hybrid arithmetical weighted (ET-LHAW) operator:

Definition 3.1 Let $X=\left\{\left(s_{1}, \alpha_{1}\right),\left(s_{2}, \alpha_{2}\right), \ldots,\left(s_{n}, \alpha_{n}\right)\right\}$ be a set of 2-tuple linguistic variables, the ET-LHAW operator of dimension $n$ is a mapping ET-LHAW: $\mathrm{R}^{n}$ $\rightarrow \mathrm{R}$ that has an associated 2-tuple linguistic weight vector $l w=\left(\left(r_{1}, g_{1}\right),\left(r_{2}, g_{2}\right), \ldots,\left(r_{n}, g_{n}\right)\right)$, denoted by $\left(s^{*}, \alpha^{*}\right)=\mathrm{ET}-L H A W_{l w, l \omega}\left(\left(s_{1}, \alpha_{1}\right),\left(s_{2}, \alpha_{2}\right), \ldots,\left(s_{n}, \alpha_{n}\right)\right)$

$$
\begin{gathered}
=\Delta\left(\sum_{j=1}^{n} \frac{\Delta^{-1}\left(r_{j}, g_{j}\right) \Delta^{-1}\left(t_{j}, \gamma_{j}\right)}{\sum_{j=1}^{n} \Delta^{-1}\left(r_{j}, g_{j}\right) \Delta^{-1}\left(\omega_{j}, \varepsilon_{j}\right)}\right) \\
S^{*} \in S, \alpha^{*} \in[-0.5,0.5),
\end{gathered}
$$

where $\Delta^{-1}\left(t_{j}, \gamma_{j}\right)$ is the $j$ th largest value of the weighted values $\Delta^{-1}\left(s_{i}, \alpha_{i}\right) \Delta^{-1}\left(\omega_{i}, \varepsilon_{i}\right) \quad(i=1,2, \ldots, n)$, $l \omega=\left(\left(\omega_{1}, \varepsilon_{1}\right),\left(\omega_{2}, \varepsilon_{2}\right), \ldots,\left(\omega_{n}, \varepsilon_{n}\right)\right)$ is the 2-tuple linguistic weight vector on $X$.

Just like any hybrid aggregation operator, in Definition 3.1, lw is a 2-tuple linguistic weight vector defined on the ordered set, while $l \omega$ is a 2-tuple linguistic weight vector defined on the element set.

Remark 3.1 If $\left(r_{i}, g_{i}\right)=\left(r_{j}, g_{j}\right)$ for all $i, j=1,2, \ldots, n$ with $i \neq j$, then the ET-LHAW operator reduces to the ET-WA operator [5], and if $\left(\omega_{i}, \varepsilon_{i}\right)=\left(\omega_{j}, \varepsilon_{j}\right)$ for all $i, j$ $=1,2, \ldots, n$ with $i \neq j$, then the ET-LHAW operator degenerates to the ET-OWA operator [23].

By extending the extended 2-tuple weighted geometric (ET-WG) operator and the extended 2-tuple ordered weighted geometric (ET-OWG) operator [26], we introduce the following extended 2-tuple linguistic hybrid geometric mean (ET-LHGM) operator:

Definition 3.2 Let $X=\left\{\left(s_{1}, \alpha_{1}\right),\left(s_{2}, \alpha_{2}\right), \ldots,\left(s_{n}, \alpha_{n}\right)\right\}$ be a set of 2-tuple linguistic variables, the ET-LHGM operator of dimension $n$ is a mapping ET-LHGM: $\mathrm{R}^{n}$ $\rightarrow \mathrm{R}$ that has an associated 2-tuple linguistic weight vector $l w=\left(\left(r_{1}, g_{1}\right),\left(r_{2}, g_{2}\right), \ldots,\left(r_{n}, g_{n}\right)\right)$, denoted by $\left(s^{\prime}, \alpha^{\prime}\right)=$ ET-LHGM $_{l w, l \omega}\left(\left(s_{1}, \alpha_{1}\right),\left(s_{2}, \alpha_{2}\right), \ldots,\left(s_{n}, \alpha_{n}\right)\right)$

$$
=\Delta\left(\prod_{j=1}^{n} \Delta^{-1}\left(t_{j}, \gamma_{j}\right)^{\frac{\Delta_{j=1}^{n} \Delta^{-1}\left(r_{j}, g_{j}\right) \Delta^{-1}\left(\omega_{j}, \varepsilon_{j}\right)}{s^{\prime} \in S,} \alpha^{\prime} \in[-0.5,0.5),}\right)
$$

where $\Delta^{-1}\left(t_{j}, \gamma_{j}\right)$ is the $j$ th largest value of the weighted values $\Delta^{-1}\left(s_{i}, \alpha_{i}\right)^{\Delta^{-1}\left(\omega_{i}, \varepsilon_{i}\right)}(i=1,2, \ldots, n), l \omega=$ $\left(\left(\omega_{1}, \varepsilon_{1}\right),\left(\omega_{2}, \varepsilon_{2}\right), \ldots,\left(\omega_{n}, \varepsilon_{n}\right)\right)$ is the 2-tuple linguistic weight vector on $X$.

Remark 3.2 If $\left(r_{i}, g_{i}\right)=\left(r_{j}, g_{j}\right)$ for all $i, j=1,2, \ldots, n$ with $i \neq j$, then the ET-LHGM operator reduces to the ET-WG operator [9], and if $\left(\omega_{i}, \varepsilon_{i}\right)=\left(\omega_{j}, \varepsilon_{j}\right)$ for all $i, j$ $=1,2, \ldots, n$ with $i \neq j$, then the ET-LHGM operator degenerates to the ET-OWG operator [26].

From the induced uncertain linguistic ordered weighted geometric (IULOWG) operator [38], Definitions 3.1 and 3.2, we further introduce the induced extended 2-tuple linguistic hybrid arithmetical weighted (IET-LHAW) operator and the induced extended 2-tuple linguistic hybrid geometric mean (IET-LHGM) operator.

Definition 3.3 Let $X=\left\{\left(s_{1}, \alpha_{1}\right),\left(s_{2}, \alpha_{2}\right), \ldots,\left(s_{n}, \alpha_{n}\right)\right\}$ be a set of 2-tuple linguistic variables, the IET-LHAW operator of dimension $n$ is a mapping IET-LHAW: $\mathrm{R}^{n} \rightarrow \mathrm{R}$ defined on the set of second arguments of two tuples $<u_{1},\left(s_{1}, \alpha_{1}\right)>,<u_{2},\left(s_{2}, \alpha_{2}\right)>$, $\ldots,<u_{n},\left(s_{n}, \alpha_{n}\right)>$ with a set of order-inducing variables $u_{i}(i=1,2, \ldots, n)$, denoted by

$$
\begin{gathered}
\left(\hat{s}^{*}, \hat{\alpha}^{*}\right)=\operatorname{IET}-\operatorname{LHAW}_{l w, l \omega}\left(<u_{1},\left(s_{1}, \alpha_{1}\right)>,<u_{2},\left(s_{2}, \alpha_{2}\right)>,\right. \\
\left.\ldots,<u_{n},\left(s_{n}, \alpha_{n}\right)>\right) \\
=\Delta\left(\sum_{j=1}^{n} \frac{\Delta^{-1}\left(r_{j}, g_{j}\right) \Delta^{-1}\left(t_{(j)}, \gamma_{(j)}\right)}{\sum_{j=1}^{n} \Delta^{-1}\left(r_{j}, g_{j}\right) \Delta^{-1}\left(\omega_{(j)}, \varepsilon_{(j)}\right)}\right) \\
\hat{s}^{*} \in S, \hat{\alpha}^{*} \in[-0.5,0.5),
\end{gathered}
$$


where $u_{(j)}$ is the $j$ th largest value of $u_{i}(j=1,2, \ldots, n)$, $\Delta^{-1}\left(t_{j}, \gamma_{j}\right)$ is the weighted value $\Delta^{-1}\left(s_{i}, \alpha_{i}\right) \Delta^{-1}\left(\omega_{i}, \varepsilon_{i}\right)$ $(i=1,2, \ldots, n), l w=\left(\left(r_{1}, g_{1}\right),\left(r_{2}, g_{2}\right), \ldots,\left(r_{n}, g_{n}\right)\right)$ is the 2-tuple linguistic weight vector on ordered set $N$ $=\{1,2, \ldots, n\}$, and $l \omega=\left(\left(\omega_{1}, \varepsilon_{1}\right),\left(\omega_{2}, \varepsilon_{2}\right), \ldots,\left(\omega_{n}, \varepsilon_{n}\right)\right)$ is the 2-tuple linguistic weight vector on $X$.

Definition 3.4 Let $X=\left\{\left(s_{1}, \alpha_{1}\right),\left(s_{2}, \alpha_{2}\right), \ldots,\left(s_{n}, \alpha_{n}\right)\right\}$ be a set of 2-tuple linguistic variables, the IET-LHGM operator of dimension $n$ is a mapping IET-LHGM: $\mathrm{R}^{n} \rightarrow \mathrm{R}$ defined on the set of second arguments of two tuples $<u_{1},\left(s_{1}, \alpha_{1}\right)>,<u_{2},\left(s_{2}, \alpha_{2}\right)>$, $\ldots,<u_{n},\left(s_{n}, \alpha_{n}\right)>$ with a set of order-inducing variables $u_{i}(i=1,2, \ldots, n)$, denoted by

$\left(\hat{s}^{\prime}, \hat{\alpha}^{\prime}\right)=\operatorname{IET}_{-L_{H G M}}\left(<u_{l},\left(s_{1}, \alpha_{1}\right)>,<u_{2},\left(s_{2}, \alpha_{2}\right)>\right.$,

$$
\begin{aligned}
&\left.\ldots,<u_{n},\left(s_{n}, \alpha_{n}\right)>\right) \\
&= \Delta\left(\prod_{j=1}^{n} \Delta^{-1}\left(t_{(j)}, \gamma_{(j)}\right)_{j=1}^{\frac{\Delta^{-1}\left(r_{j}, g_{j}\right)}{\sum_{j} \Delta^{-1}\left(r_{j}, g_{j}\right) \Delta^{-1}\left(\omega_{(j)}, \varepsilon_{(j)}\right)}}\right) \\
& \hat{s}^{\prime} \in S, \hat{\alpha}^{\prime} \in[-0.5,0.5),
\end{aligned}
$$

where $u_{(j)}$ is the $j$ th largest value of $u_{i}(j=1,2, \ldots, n)$, $\Delta^{-1}\left(t_{i}, \gamma_{i}\right)$ is the weighted value $\Delta^{-1}\left(s_{i}, \alpha_{i}\right)^{\Delta^{-1}\left(\omega_{i}, \varepsilon_{i}\right)}(i=1$, $2, \ldots, n), l w=\left(\left(r_{1}, g_{1}\right),\left(r_{2}, g_{2}\right), \ldots,\left(r_{n}, g_{n}\right)\right)$ is the 2-tuple linguistic weight vector on ordered set $N=\{1,2, \ldots, n\}$, and $l \omega=\left(\left(\omega_{1}, \varepsilon_{1}\right),\left(\omega_{2}, \varepsilon_{2}\right), \ldots,\left(\omega_{n}, \varepsilon_{n}\right)\right)$ is the 2-tuple linguistic weight vector on $X$.

However, if there is a tie between $<u_{i},\left(s_{i}, \alpha_{i}\right)>$ and $<u_{j},\left(s_{j}, \alpha_{j}\right)>$ with respect to order inducing variables such that $u_{i}=u_{j}$. In this case, $\mathrm{Xu}$ [39] presented a policy that is to replace the argument component of each of $<u_{i},\left(s_{i}, \alpha_{i}\right)>$ and $<u_{j},\left(s_{j}, \alpha_{j}\right)>$ by their average $\left(\left(s_{i}, \alpha_{i}\right) \oplus\left(s_{j}, \alpha_{j}\right)\right) / 2$. If $k$ items are tied, we replace these by $k$ replicas of their average.

Remark 3.3 If $u_{i}=\Delta^{-1}\left(s_{i}, \alpha_{i}\right) \Delta^{-1}\left(\omega_{i}, \varepsilon_{i}\right)$, then the IET-LHAW operator degenerates to the ET-LHAW operator, and if $u_{i}=\Delta^{-1}\left(s_{i}, \alpha_{i}\right)^{\Delta^{-1}\left(\omega_{i}, \varepsilon_{i}\right)}$, the IET-LHGM operator reduces to the ET-LHGM operator. Thus, the ET-LHAW and ET-LHGM operators can be seen a special case of the IET-LHAW and IET-LHGM operators, respectively.
In the following, we study some desirable properties of these four operators.

Proposition 3.1 (Commutativity) Let $X=\left\{\left(s_{1}, \alpha_{1}\right),\left(s_{2}\right.\right.$, $\left.\left.\alpha_{2}\right), \ldots,\left(s_{n}, \alpha_{n}\right)\right\}$ be a set of 2-tuple linguistic variables, and $\left(s_{i}^{\prime}, \alpha_{i}^{\prime}\right)(i=1,2, \ldots, n)$ be a permutation of $\left(s_{i}, \alpha_{i}\right)$. Then

$$
\begin{aligned}
& \operatorname{IET}-L H A W_{l w, l \omega}\left(<u_{1},\left(s_{1}, \alpha_{1}\right)>, \ldots,<u_{n},\left(s_{n}, \alpha_{n}\right)>\right)
\end{aligned}
$$

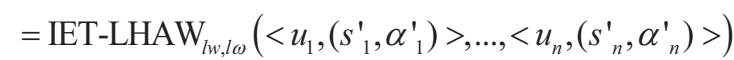

$$
\begin{aligned}
& \text { and }
\end{aligned}
$$

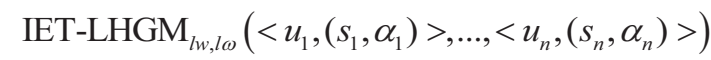

$$
\begin{aligned}
& =\operatorname{IET}_{-L_{H G M}}{ }_{l w, l \omega}\left(<u_{1},\left(s_{1}^{\prime}, \alpha_{1}^{\prime}\right)>, \ldots,<u_{n},\left(s_{n}^{\prime}, \alpha_{n}^{\prime}\right)>\right) \text {. }
\end{aligned}
$$

Corollary 3.1 Let $X=\left\{\left(s_{1}, \alpha_{1}\right),\left(s_{2}, \alpha_{2}\right), \ldots,\left(s_{n}, \alpha_{n}\right)\right\}$ be a set of 2-tuple linguistic variables, and $\left(s_{i}^{\prime}, \alpha_{i}^{\prime}\right)$ $(i=1,2, \ldots, n)$ be a permutation of $\left(s_{i}, \alpha_{i}\right)$. Then

$$
\begin{aligned}
& \text { ET-LHAW }_{l w, l \omega}\left(\left(s_{1}, \alpha_{1}\right),\left(s_{2}, \alpha_{2}\right), \ldots,\left(s_{n}, \alpha_{n}\right)\right) \\
& =\text { ET-LHAW }_{l w, l \omega}\left(\left(s_{1}^{\prime}, \alpha_{1}^{\prime}\right),\left(s_{2}^{\prime}, \alpha_{2}^{\prime}\right), \ldots,\left(s_{n}^{\prime}, \alpha_{n}^{\prime}\right)\right)
\end{aligned}
$$
and

$$
\begin{aligned}
& \operatorname{ET}-L H G M_{l w, l \omega}\left(\left(s_{1}, \alpha_{1}\right),\left(s_{2}, \alpha_{2}\right), \ldots,\left(s_{n}, \alpha_{n}\right)\right) \\
& =\operatorname{ET}-L H G M_{l w, l \omega}\left(\left(s_{1}^{\prime}, \alpha_{1}^{\prime}\right),\left(s_{2}^{\prime}, \alpha_{2}^{\prime}\right), \ldots,\left(s_{n}^{\prime}, \alpha_{n}^{\prime}\right)\right) .
\end{aligned}
$$

Proposition 3.2 (Monotonicity) Let $X=\left\{\left(s_{1}, \alpha_{1}\right),\left(s_{2}\right.\right.$, $\left.\left.\alpha_{2}\right), \ldots,\left(s_{n}, \alpha_{n}\right)\right\}$ and $X^{\prime}=\left\{\left(s_{1}^{\prime}, \alpha_{1}^{\prime}\right),\left(s_{2}^{\prime}, \alpha_{2}^{\prime}\right), \ldots,\left(s_{n}^{\prime}, \alpha_{n}^{\prime}\right)\right\}$ be two sets of 2-tuple linguistic variables. If $\left(s_{i}, \alpha_{i}\right) \leq$ $\left(s_{i}^{\prime}, \alpha_{i}^{\prime}\right)$ for each $i=1,2, \ldots, n$, then

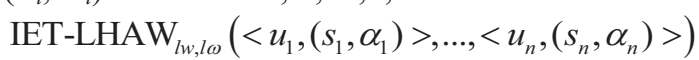

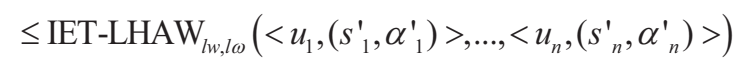

$$
\begin{aligned}
& \text { and }
\end{aligned}
$$

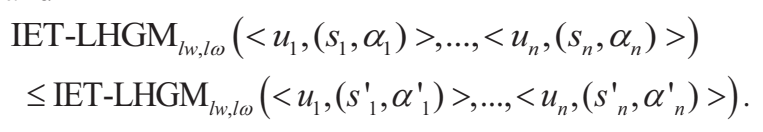

Corollary 3.2 Let $X=\left\{\left(s_{1}, \alpha_{1}\right),\left(s_{2}, \alpha_{2}\right), \ldots,\left(s_{n}, \alpha_{n}\right)\right\}$ and $X^{\prime}=\left\{\left(s_{1}^{\prime}, \alpha_{1}^{\prime}\right),\left(s_{2}^{\prime}, \alpha_{2}^{\prime}\right), \ldots,\left(s_{n}^{\prime}, \alpha_{n}^{\prime}\right)\right\}$ be two sets of 2-tuple linguistic variables. If $\left(s_{\mathrm{i}}, \alpha_{\mathrm{i}}\right) \leq\left(s_{i}^{\prime}, \alpha_{i}^{\prime}\right)$ for each $i=1,2, \ldots, n$, then

$$
\text { ET-LHAW }_{l w, l \omega}\left(\left(s_{1}, \alpha_{1}\right),\left(s_{2}, \alpha_{2}\right), \ldots,\left(s_{n}, \alpha_{n}\right)\right)
$$$$
\leq \text { ET-LHAW }_{l w, l \omega}\left(\left(s_{1}^{\prime}, \alpha_{1}^{\prime}\right),\left(s_{2}^{\prime}, \alpha_{2}^{\prime}\right), \ldots,\left(s_{n}^{\prime}, \alpha_{n}^{\prime}\right)\right)
$$
and

$\operatorname{ET}_{-L H G M}{ }_{l w, l \omega}\left(\left(s_{1}, \alpha_{1}\right),\left(s_{2}, \alpha_{2}\right), \ldots,\left(s_{n}, \alpha_{n}\right)\right)$

$$
\leq \operatorname{ET}_{-L_{H G M}}{ }_{l w, l \omega}\left(\left(s_{1}^{\prime}, \alpha_{1}^{\prime}\right),\left(s_{2}^{\prime}, \alpha_{2}^{\prime}\right), \ldots,\left(s_{n}^{\prime}, \alpha_{n}^{\prime}\right)\right) \text {. }
$$

Proposition 3.3 (Idempotency) Let $X=\left\{\left(s_{1}, \alpha_{1}\right),\left(s_{2}\right.\right.$, $\left.\left.\alpha_{2}\right), \ldots,\left(s_{n}, \alpha_{n}\right)\right\}$ be a set of 2-tuple linguistic variables. If $\left(s_{i}, \alpha_{i}\right)=(s, \alpha)$ for each $i=1,2, \ldots, n$, then $\operatorname{IET}_{-L_{H A W}}\left(<u_{l},\left(s_{1}, \alpha_{1}\right)>, \ldots,<u_{n},\left(s_{n}, \alpha_{n}\right)>\right)=(s, \alpha)$ and 
$\operatorname{IET}-L H G M_{l w, l \omega}\left(<u_{1},\left(s_{1}, \alpha_{1}\right)>, \ldots,<u_{n},\left(s_{n}, \alpha_{n}\right)>\right)=(s, \alpha)$.

Corollary 3.3 Let $X=\left\{\left(s_{1}, \alpha_{1}\right),\left(s_{2}, \alpha_{2}\right), \ldots,\left(s_{n}, \alpha_{n}\right)\right\}$ be a set of 2-tuple linguistic variables. If $\left(s_{i}, \alpha_{i}\right)=(s, \alpha)$ for each $i=1,2, \ldots, n$, then

$$
\begin{aligned}
& \operatorname{ET}_{\text {and }} \operatorname{LHAW}_{l w, l \omega}\left(\left(s_{1}, \alpha_{1}\right),\left(s_{2}, \alpha_{2}\right), \ldots,\left(s_{n}, \alpha_{n}\right)\right)=(s, \alpha) \\
& \operatorname{ET}_{-L_{H G G}}{ }_{l w, l \omega}\left(\left(s_{1}, \alpha_{1}\right),\left(s_{2}, \alpha_{2}\right), \ldots,\left(s_{n}, \alpha_{n}\right)\right)=(s, \alpha) .
\end{aligned}
$$

Proposition 3.4 (Boundary) Let $X=\left\{\left(s_{1}, \alpha_{1}\right), \quad\left(s_{2}\right.\right.$, $\left.\left.\alpha_{2}\right), \ldots,\left(s_{n}, \alpha_{n}\right)\right\}$ be a set of 2-tuple linguistic variables, then

$$
\begin{aligned}
& \min \left(\left(s_{1}, \alpha_{1}\right), \ldots,\left(s_{n}, \alpha_{n}\right)\right) \\
& \leq \operatorname{IET}-\operatorname{LHAW}_{l w, l \omega}\left(<u_{1},\left(s_{1}, \alpha_{1}\right)>, \ldots,<u_{n},\left(s_{n}, \alpha_{n}\right)>\right) \\
& \leq \max \left(\left(s_{1}, \alpha_{1}\right), \ldots,\left(s_{n}, \alpha_{n}\right)\right) \\
& \text { and } \\
& \min \left(\left(s_{1}, \alpha_{1}\right), \ldots,\left(s_{n}, \alpha_{n}\right)\right) \\
& \leq \operatorname{IET}-\operatorname{LHGM}_{l w, l \omega}\left(<u_{1},\left(s_{1}, \alpha_{1}\right)>, \ldots,<u_{n},\left(s_{n}, \alpha_{n}\right)>\right) \\
& \leq \max \left(\left(s_{1}, \alpha_{1}\right), \ldots,\left(s_{n}, \alpha_{n}\right)\right) .
\end{aligned}
$$

Corollary 3.4 Let $X=\left\{\left(s_{1}, \alpha_{1}\right),\left(s_{2}, \alpha_{2}\right), \ldots,\left(s_{n}, \alpha_{n}\right)\right\}$ be a set of 2-tuple linguistic variables, then

$$
\begin{aligned}
& \min \left(\left(s_{1}, \alpha_{1}\right), \ldots,\left(s_{n}, \alpha_{n}\right)\right) \\
\leq & \operatorname{ET}-\operatorname{LHAW}_{l w}, l \omega \\
\leq & \left(\left(s_{1}, \alpha_{1}\right),\left(s_{2}, \alpha_{2}\right), \ldots,\left(s_{n}, \alpha_{n}\right)\right) \\
\leq & \max \left(\left(s_{1}, \alpha_{1}\right), \ldots,\left(s_{n}, \alpha_{n}\right)\right)
\end{aligned}
$$

and

$$
\begin{aligned}
& \min \left(\left(s_{1}, \alpha_{1}\right), \ldots,\left(s_{n}, \alpha_{n}\right)\right) \\
& \leq \operatorname{ET}-\operatorname{LHGM}_{l w, l \omega}\left(\left(s_{1}, \alpha_{1}\right),\left(s_{2}, \alpha_{2}\right), \ldots,\left(s_{n}, \alpha_{n}\right)\right) \\
& \leq \max \left(\left(s_{1}, \alpha_{1}\right), \ldots,\left(s_{n}, \alpha_{n}\right)\right) .
\end{aligned}
$$

\section{The models for the optimal linguistic weight vectors}

Based on distance deviation method, TOPSIS method [40] and mean deviation method, we research the models for the optimal linguistic weight vectors.

Consider a multi-attribute group decision-making problem, in which both the weights and the attribute preference values take the form of linguistic variables. Let $A=\left\{a_{1}, a_{2}, \ldots, a_{m}\right\}$ be the set of alternatives, $C=\left\{c_{1}\right.$, $\left.c_{2}, \ldots, c_{n}\right\}$ be the set of attributes, and $E=\left\{e_{1}, e_{2}, \ldots, e_{q}\right\}$ be the set of the experts. Assume that $\tilde{Q}^{k}=\left(q_{i j}^{k}\right)_{m \times n}$ is the decision matrix, where $q_{i j}^{k} \in S$ is a preference value, which takes the form of linguistic variables, given by the expert $e_{k}$, for the alternative $a_{i} \in A$ w.r.t. the attribute $c_{j} \in C$.

Let the linguistic weight vectors be evaluated using the linguistic term set $Z$. For example, $Z$ can be defined as

$Z=\left\{z_{1}\right.$ : extremely unimportant, $z_{2}$ : very unimportant, $z_{3}$ :unimportant; $z_{4}$ : fair, $z_{5}$ : important, $z_{6}$ : very important, $z_{7}$ : extremely important $\}$.

Transform respectively the linguistic decision matrix $\tilde{Q}^{k}=\left(q_{i j}^{k}\right)_{m \times n}$, the interval linguistic weight vector

$$
l \bar{w}_{E}^{j}=\left(\left[z_{l e_{1}}^{j}, z_{r e_{1}}^{j}\right],\left[z_{l e_{2}}^{j}, z_{r e_{2}}^{j}\right], \ldots,\left[z_{l e_{q}}^{j}, z_{r e_{q}}^{j}\right]\right)
$$

on the expert set $E$ for each attribute $c_{j}(j=1,2, \ldots, n)$ and the interval linguistic weight vector

$$
l \bar{w}_{Q}=\left(\left[z_{l 1}, z_{r 1}\right],\left[z_{l 2}, z_{r 2}\right], \ldots,\left[z_{l q}, z_{r q}\right]\right)
$$

on ordered set $Q=\{1,2, \ldots, q\}$ into the 2-tuple linguistic decision matrix $\tilde{Q}^{k}=\left(\left(q_{i j}^{k}, 0\right)\right)_{m \times n}$, the 2-tuple interval linguistic weight vector $l \bar{\omega}_{E}^{j}=\left(\left[\left(z_{l e_{1}}^{j}, 0\right),\left(z_{r e_{1}}^{j}, 0\right)\right],\left[\left(z_{l e_{2}}^{j}, 0\right),\left(z_{r e_{2}}^{j}, 0\right)\right], \ldots,\left[\left(z_{l e_{q}}^{j}, 0\right),\left(z_{r e_{q}}^{j}, 0\right)\right]\right)$ and the 2-tuple interval linguistic weight vector $l \bar{w}_{Q}=\left(\left[\left(z_{l 1}, 0\right),\left(z_{r 1}, 0\right)\right],\left[\left(z_{l 2}, 0\right),\left(z_{r 2}, 0\right)\right], \ldots,\left[\left(z_{l q}, 0\right),\left(z_{r q}, 0\right)\right]\right)$, with $z_{l e_{k}}^{j}, z_{r e_{k}}^{j}, z_{l k}, z_{r k} \in Z$ for each $k \in Q$.

\subsection{The model for the weight vector on the expert set}

For each attribute $c_{j}(j=1,2, \ldots, n)$, calculate the distance $d_{k}^{j}$ between the expert $e_{k}$ and each expert in $E \backslash\left\{e_{k}\right\}$ as follows:

$$
d_{k}^{j}=\sum_{l=1, l \neq k}^{q} \sum_{i=1}^{m}\left|\Delta^{-1}\left(q_{i j}^{k}, 0\right)-\Delta^{-1}\left(q_{i j}^{l}, 0\right)\right| .
$$

Based on distance deviation method, we build the following model for the optimal linguistic weight vector on the expert set $E$ for the attribute $c_{j}$ $(j=1,2, \ldots, n)$.

$$
\begin{gathered}
\min \sum_{k=1}^{q} d_{k}^{j} \frac{\Delta^{-1}\left(z_{e_{k}}^{j}, \alpha_{e_{k}}^{j}\right)}{\sum_{l=1}^{q} \Delta^{-1}\left(z_{e_{l}}^{j}, \alpha_{e_{l}}^{j}\right)} \\
\text { s.t. } \Delta^{-1}\left(z_{e_{k}}^{j}, \alpha_{e_{k}}^{j}\right) \in\left[\Delta^{-1}\left(z_{l_{k}}^{j}, 0\right), \Delta^{-1}\left(z_{r_{k}}^{j}, 0\right)\right], e_{k} \in E .
\end{gathered}
$$

It is equivalent to

$$
\text { s.t. }\left\{\begin{array}{c}
\min \lambda \\
\sum_{k=1}^{q} d_{k}^{j} \Delta^{-1}\left(z_{e_{k}}^{j}, \alpha_{e_{k}}^{j}\right)-\lambda \sum_{k=1}^{q} \Delta^{-1}\left(z_{e_{k}}^{j}, \alpha_{e_{k}}^{j}\right)=0 \\
\Delta^{-1}\left(z_{e_{k}}^{j}, \alpha_{e_{k}}^{j}\right) \in\left[\Delta^{-1}\left(z_{l e_{k}}^{j}, 0\right), \Delta^{-1}\left(z_{r e_{k}}^{j}, 0\right)\right], e_{k} \in E
\end{array} .\right.
$$

The optimal linguistic weights obtained by this 
method have the following desirable characteristics: the closer an expert's evaluation values are to other experts', the larger the weight measure is. This can avoid the unduly high or low evaluation values induced by experts' limited knowledge or expertise.

\subsection{The model for the weight vector on the} ordered set $Q$

Consider the linguistic weights on the ordered set $Q$ $=\{1,2, \ldots, q\}$ for each pair $(i, j)(i=1,2, . ., m ; j=1,2, \ldots, n)$. Calculate the arithmetic averaging value $d_{i j}$ for each pair $(i, j)(i=1,2, . ., m ; j=1,2, \ldots, n)$ as follows:

$$
d_{i j}=\frac{1}{q} \sum_{k=1}^{q} \Delta^{-1}\left(q_{i j}^{k}, 0\right)
$$

and compute the distance $d_{i j}^{k}$ between $\Delta^{-1}\left(\tilde{q}_{i j}^{k}, 0\right)$ and $d_{i j}$ as follows:

$$
d_{i j}^{k}=\left|\Delta^{-1}\left(q_{i j}^{k}, 0\right)-d_{i j}\right| \quad \forall k \in Q .
$$

Then, reorder $d_{i j}^{k}(k \in Q)$ in decreasing order $d_{i j}^{(1)} \geq d_{i j}^{(2)} \geq \ldots \geq d_{i j}^{(q)}$. Based on mean deviation method, we establish the following model for the optimal linguistic weight vector on the ordered set $Q$ for each pair $(i, j)(i=1,2, . ., m ; j=1,2, \ldots, n)$.

$$
\min \sum_{k=1}^{q} d_{i j}^{(k)} \frac{\Delta^{-1}\left(z_{k}^{i j}, \alpha_{k}^{i j}\right)}{\sum_{l=1}^{q} \Delta^{-1}\left(z_{l}^{i j}, \alpha_{l}^{i j}\right)}
$$

s.t. $\Delta^{-1}\left(z_{k}^{i j}, \alpha_{k}^{i j}\right) \in\left[\Delta^{-1}\left(z_{l k}, 0\right), \Delta^{-1}\left(z_{r k}, 0\right)\right], k \in Q$.

It is equivalent to

$$
\text { s.t. }\left\{\begin{array}{c}
\min \lambda \\
\sum_{k=1}^{q} d_{i j}^{(k)} \Delta^{-1}\left(z_{k}^{i j}, \alpha_{k}^{i j}\right)-\lambda \sum_{k=1}^{q} \Delta^{-1}\left(z_{k}^{i j}, \alpha_{k}^{i j}\right)=0 \\
\Delta^{-1}\left(z_{k}^{i j}, \alpha_{k}^{i j}\right) \in\left[\Delta^{-1}\left(z_{l k}, 0\right), \Delta^{-1}\left(z_{r k}, 0\right)\right], k \in Q
\end{array}\right.
$$

If there is more than one $d_{i j}$ equal, then we reorder their positions according to their subscripts in increasing order.

\subsection{The model for the weight vector on the attribute set}

Based on TOPSIS method [40], we introduce the following model for the optimal linguistic weight vector on attribute set $C$.

Let $\tilde{Q}=\left(\left(q_{i j}, \alpha_{i j}\right)\right)_{m \times n}$ be the comprehensive 2-tuple linguistic decision matrix. Transform the interval linguistic weight vector

$$
l \bar{\omega}_{C}=\left(\left[z_{l c_{1}}, z_{r c_{1}}\right],\left[z_{l c_{2}}, z_{r c_{2}}\right], \ldots,\left[z_{l c_{n}}, z_{r c_{n}}\right]\right)
$$

on attribute set $C$ and the interval linguistic weight vector

$$
l \bar{w}_{N}^{i}=\left(\left[z_{l 1}, z_{r 2}\right],\left[z_{l 1}, z_{r 2}\right], \ldots,\left[z_{l n}, z_{r n}\right]\right)
$$

on ordered set $N$ for each $i=1,2, \ldots, m$ into the 2-tuple interval linguistic weight vectors

$l \bar{\omega}_{C}=\left(\left[\left(z_{l c_{1}}, 0\right),\left(z_{r c_{1}}, 0\right)\right],\left[\left(z_{l c_{2}}, 0\right),\left(z_{r c_{2}}, 0\right)\right], \ldots,\left[\left(z_{l c_{n}}, 0\right),\left(z_{r c_{n}}, 0\right)\right]\right)$ and

$l \bar{w}_{N}^{i}=\left(\left[\left(z_{l 1} 0\right),\left(z_{r 1} 0\right)\right],\left[\left(z_{l 2} 0\right),\left(z_{r 2} 0\right)\right], \ldots,\left[\left(z_{l n} 0\right),\left(z_{r n} 0\right)\right]\right)$, with $z_{l c_{j}}, z_{r c_{j}}, z_{l j}, z_{r j} \in Z$ for each $j \in N$, respectively.

Let $\tilde{R}^{+}=\left(\tilde{r}_{1}^{+}, \tilde{r}_{2}^{+}, \ldots, \tilde{r}_{n}^{+}\right)$and $\tilde{R}^{-}=\left(\tilde{r}_{1}^{-}, \tilde{r}_{2}^{-}, \ldots, \tilde{r}_{n}^{-}\right)$be the positive and negative ideal vectors, respectively, where $\tilde{r}_{j}^{+}=\max _{1 \leq i \leq m}\left(q_{i j}, \alpha_{i j}\right)$ and $\tilde{r}_{j}^{-}=\min _{1 \leq i \leq m}\left(q_{i j}, \alpha_{i j}\right)$ for all $j=1,2, \ldots, n$.

For each $i=1,2, \ldots, m$, calculate the distances $d_{i}^{+}$and $d_{i}^{-}$as follows:

$$
\begin{aligned}
& d_{i j}^{+}=\left|\Delta^{-1}\left(q_{i j}, \alpha_{i j}\right)-\Delta^{-1}\left(\tilde{r}_{j}^{+}\right)\right|, \\
& d_{i j}^{-}=\left|\Delta^{-1}\left(q_{i j}, \alpha_{i j}\right)-\Delta^{-1}\left(\tilde{r}_{j}^{-}\right)\right| .
\end{aligned}
$$

Since all alternatives are non inferior, we build the following model for the optimal linguistic weight vector on the attribute set $C$.

$$
\begin{gathered}
\min \sum_{i=1}^{m} \sum_{j=1}^{n} \frac{d_{i j}^{+}}{d_{i j}^{+}+d_{i j}^{-}} \frac{\Delta^{-1}\left(z_{c_{j}}, \alpha_{c_{j}}\right)}{\sum_{j=1}^{n} \Delta^{-1}\left(z_{c_{j}}, \alpha_{c_{j}}\right)} \\
\text { s.t. } \Delta^{-1}\left(z_{c_{j}}, \alpha_{c_{j}}\right) \in\left[\Delta^{-1}\left(z_{l c_{j}}, 0\right), \Delta^{-1}\left(z_{r c_{j}}, 0\right)\right], c_{j} \in C .
\end{gathered}
$$

It is equivalent to

$$
\text { s.t. }\left\{\begin{array}{c}
\sum_{i=1}^{m} \sum_{j=1}^{n} \frac{d_{i j}^{+}}{d_{i j}^{+}+d_{i j}^{-}} \Delta^{-1}\left(z_{c_{j}}, \alpha_{c_{j}}\right)-\lambda \sum_{j=1}^{n} \Delta^{-1}\left(z_{c_{j}}, \alpha_{c_{j}}\right)=0 \\
\Delta^{-1}\left(z_{c_{j}}, \alpha_{c_{j}}\right) \in\left[\Delta^{-1}\left(z_{l c_{j}}, 0\right), \Delta^{-1}\left(z_{r c_{j}}, 0\right)\right], c_{j} \in C
\end{array} .\right.
$$

\subsection{The model for the weight vector on the ordered set $N$}

Similar to the model for the optimal linguistic weight vector on the ordered set $Q$, we introduce the following model for the optimal linguistic weight vector on the ordered set $N$ for each $i=1,2, \ldots, m$.

For each $i=1,2, \ldots, m$, calculate the arithmetic average

$$
d_{j}=\frac{1}{n} \sum_{i=1}^{m} \Delta^{-1}\left(q_{i j}, \alpha_{i j}\right),
$$

and compute the distance $d^{\prime}{ }_{i j}$ between $\Delta^{-1}\left(q_{i j}, \alpha_{i j}\right)$ 
and $d_{j}$ as follows:

$$
d_{i j}^{\prime}=\left|\Delta^{-1}\left(q_{i j}, \alpha_{i j}\right)-d_{j}\right| .
$$

Then, reorder $d_{i j}^{\prime}$ in decreasing order $d_{i(1)}^{\prime} \geq d_{i(2)}^{\prime} \geq \ldots \geq d_{i(n)}^{\prime}$. If there is more than one $d_{i j}$ equal, then we reorder their positions according to their subscripts in increasing order.

Based on mean deviation method, we established the following model for the optimal linguistic weight vector on the ordered set $N$ for each $i=1,2, \ldots, m$.

$$
\min \sum_{j=1}^{n} d_{i(j)}^{\prime} \frac{\Delta^{-1}\left(z_{j}^{i}, \alpha_{j}^{i}\right)}{\sum_{j=1}^{n} \Delta^{-1}\left(z_{j}^{i}, \alpha_{j}^{i}\right)}
$$

$$
\text { s.t. } \Delta^{-1}\left(z_{j}, \alpha_{j}\right) \in\left[\Delta^{-1}\left(z_{l j}, 0\right), \Delta^{-1}\left(z_{r j}, 0\right)\right], j \in N \text {. }
$$

It is equivalent to

$$
\text { s.t. }\left\{\begin{array}{c}
\min \lambda \\
\sum_{j=1}^{n} d^{\prime}{ }_{i(j)} \Delta^{-1}\left(z_{j}^{i}, \alpha_{j}^{i}\right)-\lambda \sum_{j=1}^{n} \Delta^{-1}\left(z_{j}^{i}, \alpha_{j}^{i}\right)=0 \\
\Delta^{-1}\left(z_{j}^{i}, \alpha_{j}^{i}\right) \in\left[\Delta^{-1}\left(z_{l j}, 0\right), \Delta^{-1}\left(z_{r j}, 0\right)\right], j \in N
\end{array} .\right.
$$

\section{A new approach to multi-attribute group decision making under linguistic environment}

This section develops an approach to multi-attribute group decision making under linguistic environment. The main decision procedure can be described as follows:

Step 1: Transform the linguistic decision matrix $\tilde{Q}^{k}=\left(q_{i j}^{k}\right)_{m \times n} \quad$ into 2-tuple linguistic decision matrix $\tilde{Q}^{k}=\left(\left(q_{i j}^{k}, 0\right)\right)_{m \times n}$, and convert the interval linguistic weight vector

$$
l \bar{\omega}_{E}^{j}=\left(\left[z_{l e_{1}}^{j}, z_{r e_{1}}^{j}\right],\left[z_{l e_{2}}^{j}, z_{r e_{2}}^{j}\right], \ldots,\left[z_{l e_{q}}^{j}, z_{r e_{q}}^{j}\right]\right)
$$

on the expert set $E$ for each attribute $c_{j}$ $(j=1,2, \ldots, n)$ and the interval linguistic weight vector

$$
l \bar{w}_{Q}=\left(\left[z_{l 1}, z_{r 2}\right],\left[z_{l 1}, z_{r 2}\right], \ldots,\left[z_{l q}, z_{r q}\right]\right)
$$

on the ordered set $Q=\{1,2, \ldots, q\}$ into the 2-tuple interval linguistic weight vectors

$$
\begin{aligned}
& l \bar{\omega}_{E}^{j}=(\left(\left[z_{l e_{1}}^{j}, 0\right),\left(z_{r e_{1}}^{j}, 0\right)\right],\left[\left(z_{l e_{2}}^{j}, 0\right),\left(z_{r e_{2}}^{j}, 0\right)\right], \\
&\left.\ldots,\left[\left(z_{l e_{q}}^{j}, 0\right),\left(z_{r e_{q}}^{j}, 0\right)\right]\right) \\
& \text { and } \\
& \qquad \bar{w}_{Q}=\left(\left[\left(z_{l 1}, 0\right),\left(z_{r 1}, 0\right)\right],\left[\left(z_{l 2}, 0\right),\left(z_{r 2}, 0\right)\right],\right. \\
&\left.\quad \ldots,\left[\left(z_{l q}, 0\right),\left(z_{r q}, 0\right)\right]\right),
\end{aligned}
$$

$$
\text { with } z_{l e_{k}}^{j}, z_{r e_{k}}^{j}, z_{l k}, z_{r k} \in Z \text { for each } k \in Q \text {. }
$$

Step 2: Utilize the model (8) to calculate the optimal linguistic weight vector on the experts set $E$ for the attribute $c_{j}(j=1,2, \ldots, n)$.

Step 3: Utilize the model (10) to calculate the optimal linguistic weight vector on the ordered set $Q$ for each pair $(i, j)(i=1,2, \ldots, m$; $j=1,2, \ldots, n)$.

Step 4: Use the IET-LHAW operator or the IET-LHGM operator to calculate the comprehensive 2-tuple linguistic decision matrix $\tilde{Q}=\left(\left(q_{i j}, \alpha_{i j}\right)\right)_{m \times n}$, where $\left(z_{e_{(k)}^{j}}^{j}, \alpha_{e_{(k)}^{j}}\right)$ $(k \in Q)$ is the optimal 2-tuple linguistic weight of the expert $e_{(k)}$ for the attribute $c_{j}$ $(j=1,2, \ldots, n)$, and $\left(z_{k}^{i j}, \alpha_{k}^{i j}\right)$ is the optimal 2-tuple linguistic weight of the $k$ th position for the pair $(i, j)(i=1,2, . ., m ; j=1,2, \ldots, n)$.

Step 5: Transform the interval linguistic weight vector

$$
l \bar{\omega}_{C}=\left(\left[z_{l c_{1}}, z_{r c_{1}}\right],\left[z_{l c_{2}}, z_{r c_{2}}\right], \ldots,\left[z_{l c_{n}}, z_{r c_{n}}\right]\right)
$$

on the attribute set $C$ and the interval linguistic weight vector

$$
l \bar{w}_{N}^{i}=\left(\left[z_{l 1}, z_{r 2}\right],\left[z_{l 1}, z_{r 2}\right], \ldots,\left[z_{l n}, z_{r n}\right]\right)
$$

on the ordered set $N$ for each $i=1,2, \ldots, m$ into the 2-tuple interval linguistic weight vectors

$$
\begin{aligned}
& l \bar{\omega}_{C}=\left(\left[\left(z_{l c_{1}}, 0\right),\left(z_{r c_{1}}, 0\right)\right],\left[\left(z_{l c_{2}}, 0\right),\left(z_{r c_{2}}, 0\right)\right]\right. \text {, } \\
& \left.\ldots,\left[\left(z_{l c_{n}}, 0\right),\left(z_{r c_{n}}, 0\right)\right]\right) \\
& l \bar{w}_{N}^{i}=\left(\left[\left(z_{l 1} 0\right),\left(z_{r 1} 0\right)\right],\left[\left(z_{l 2} 0\right),\left(z_{r 2} 0\right)\right], \ldots,\right. \\
& \left.\left[\left(z_{l n} 0\right),\left(z_{r n} 0\right)\right]\right) \text {, } \\
& \text { with } z_{l c_{j}}, z_{r c_{j}}, z_{l j}, z_{r j} \in Z \text { for each } j \in N \text {. }
\end{aligned}
$$

Step 6: Utilize the model (12) to calculate the optimal linguistic weight vector on the attribute set $C$.

Step 7: Utilize the model (14) to calculate the optimal linguistic weight vector on the ordered set $Q$ for each $i=1,2, \ldots, m$.

Step 8: Again use the IET-LHAW operator or the IET-LHGM operator to compute the comprehensive 2-tuple linguistic values $\left(s_{i}, \alpha_{i}\right)(i=1,2, \ldots, m)$, where $\left(z_{c_{(j)}}, \alpha_{c_{(j)}}\right)(j \in N)$ is the optimal 2-tuple linguistic weight of the attribute $c_{(j)}$ and $\left(z_{j}^{i}, \alpha_{j}^{i}\right)$ is the optimal 2-tuple linguistic weight of the $j$ th position 
for each $i=1,2, \ldots, m$.

Step 9: According to the comprehensive 2-tuple linguistic values $\left(s_{i}, \alpha_{i}\right)(i=1,2, \ldots, m)$ and the relationship between 2-tuple linguistic arguments [5], select the best one(s).

\section{A practical example}

Let us suppose there is an investment company, which wants to invest a sum of money in the best option (adapted from Ref. [41]). There is a panel with five possible alternatives $A=\left\{a_{1}, a_{2}, a_{3}, a_{4}, a_{5}\right\}$ to invest the money: $a_{1}$ is a car company; $a_{2}$ is a food company; $a_{3}$ is a computer company; $a_{4}$ is an arms company; $a_{5}$ is a TV company. The investment company must take a decision according to the following four attributes $C=\left\{c_{1}, c_{2}, c_{3}, c_{4}\right\}: c_{1}$ is the risk analysis; $c_{2}$ is the growth analysis; $c_{3}$ is the social-political impact analysis; $c_{4}$ is the environmental impact analysis. The five possible alternatives $a_{i}(i=1,2,3,4,5)$ are to be evaluated using the linguistic term set $S=\left\{s_{1}\right.$ : extremely poor, $s_{2}$ : very poor, $s_{3}$ : poor, $s_{4}$ : fair, $s_{5}$ : good, $s_{6}$ : very good, $s_{7}$ : extremely good $\}$ by three experts $E=\left\{e_{1}, e_{2}, e_{3}\right\}$ under the above four attributes, and construct the decision matrices $\tilde{Q}^{k}=\left(q_{i j}^{k}\right)_{5 \times 4}(k=1,2,3)$ as follows:

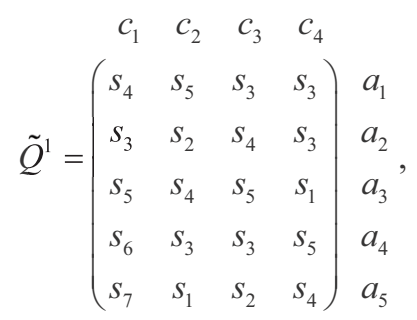

$$
\begin{aligned}
& \begin{array}{llll}
c_{1} & c_{2} & c_{3} & c_{4}
\end{array}
\end{aligned}
$$

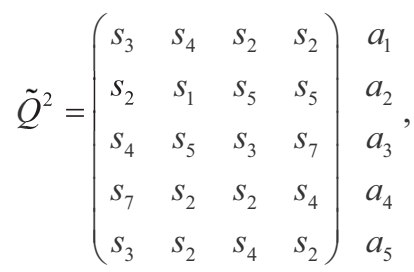

$$
\begin{aligned}
& \begin{array}{llll}
c_{1} & c_{2} & c_{3} & c_{4}
\end{array}
\end{aligned}
$$

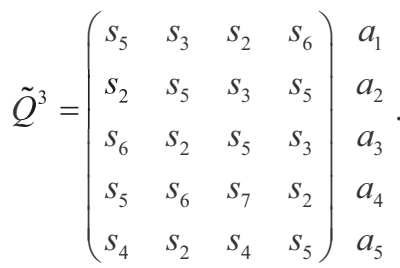

The weight vectors on the expert set $E$, on the ordered set $Q=\{1,2,3\}$, on the attribute set $C$ and on the ordered set $N=\{1,2,3,4\}$ are all evaluated using the linguistic term set $Z=\left\{z_{1}\right.$ : extremely unimportant, $z_{2}$ : very unimportant, $z_{3}$ : unimportant; $z_{4}$ : fair, $z_{5}$ : important, $z_{6}$ : very important, $z_{7}$ : extremely important\}. Assume that the experts' weight vectors are given by

$$
\begin{aligned}
& l \bar{\omega}_{e_{1}}=\left(\left[z_{2}, z_{3}\right],\left[z_{6}, z_{7}\right],\left[z_{2}, z_{4}\right],\left[z_{5}, z_{6}\right]\right), \\
& l \bar{\omega}_{e_{2}}=\left(\left[z_{5}, z_{6}\right],\left[z_{1}, z_{3}\right],\left[z_{2}, z_{4}\right],\left[z_{4}, z_{5}\right]\right), \\
& l \bar{\omega}_{e_{3}}=\left(\left[z_{3}, z_{4}\right],\left[z_{4}, z_{5}\right],\left[z_{2}, z_{3}\right],\left[z_{3}, z_{5}\right]\right),
\end{aligned}
$$

and the weight vector on the ordered set $Q$ is defined by $l \bar{w}_{Q}=\left(\left[z_{2}, z_{3}\right],\left[z_{3}, z_{4}\right],\left[z_{5}, z_{6}\right]\right)$.

According to the judgment of the three experts, the weights of attributes are known as

$$
l \bar{\omega}_{C}=\left(\left[z_{5}, z_{6}\right],\left[z_{4}, z_{6}\right],\left[z_{3}, z_{4}\right],\left[z_{5}, z_{7}\right]\right),
$$

and the weights of the ordered positions are known as $l \bar{w}_{N}^{i}=\left(\left[z_{1}, z_{2}\right],\left[z_{2}, z_{3}\right],\left[z_{3}, z_{4}\right],\left[z_{5}, z_{6}\right]\right)$ for each $i=1,2$, $3,4,5$. In the following, we can utilize the proposed procedure to get the most desirable investment company(s).

Step 1: Transform the linguistic decision matrices $\tilde{Q}^{k}=\left(q_{i j}^{k}\right)_{m \times n} \quad(k=1,2,3)$ into the 2-tuple linguistic decision matrices

$$
\begin{gathered}
\tilde{Q}^{1}=\left(\begin{array}{llll}
\left(s_{4}, 0\right) & \left(s_{5}, 0\right) & \left(s_{3}, 0\right) & \left(s_{3}, 0\right) \\
\left(s_{3}, 0\right) & \left(s_{2}, 0\right) & \left(s_{4}, 0\right) & \left(s_{3}, 0\right) \\
\left(s_{5}, 0\right) & \left(s_{4}, 0\right) & \left(s_{5}, 0\right) & \left(s_{1}, 0\right) \\
\left(s_{6}, 0\right) & \left(s_{3}, 0\right) & \left(s_{3}, 0\right) & \left(s_{5}, 0\right) \\
\left(s_{7}, 0\right) & \left(s_{1}, 0\right) & \left(s_{2}, 0\right) & \left(s_{4}, 0\right)
\end{array}\right), \\
\tilde{Q}^{2}=\left(\begin{array}{llll}
\left(s_{3}, 0\right) & \left(s_{4}, 0\right) & \left(s_{2}, 0\right) & \left(s_{2}, 0\right) \\
\left(s_{2}, 0\right) & \left(s_{1}, 0\right) & \left(s_{5}, 0\right) & \left(s_{5}, 0\right) \\
\left(s_{4}, 0\right) & \left(s_{5}, 0\right) & \left(s_{3}, 0\right) & \left(s_{7}, 0\right) \\
\left(s_{7}, 0\right) & \left(s_{2}, 0\right) & \left(s_{2}, 0\right) & \left(s_{4}, 0\right) \\
\left(s_{3}, 0\right) & \left(s_{2}, 0\right) & \left(s_{4}, 0\right) & \left(s_{2}, 0\right)
\end{array}\right), \\
\tilde{Q}^{3}=\left(\begin{array}{llll}
\left(s_{5}, 0\right) & \left(s_{3}, 0\right) & \left(s_{2}, 0\right) & \left(s_{6}, 0\right) \\
\left(s_{2}, 0\right) & \left(s_{5}, 0\right) & \left(s_{3}, 0\right) & \left(s_{5}, 0\right) \\
\left(s_{6}, 0\right) & \left(s_{2}, 0\right) & \left(s_{5}, 0\right) & \left(s_{3}, 0\right) \\
\left(s_{5}, 0\right) & \left(s_{6}, 0\right) & \left(s_{7}, 0\right) & \left(s_{2}, 0\right) \\
\left(s_{4}, 0\right) & \left(s_{2}, 0\right) & \left(s_{4}, 0\right) & \left(s_{5}, 0\right)
\end{array}\right) .
\end{gathered}
$$

Transform the interval linguistic weight vectors on the expert set $E$ for each attribute $c_{j}(j=1,2,3,4)$ into the 2-tuple linguistic weight vectors

$$
l \bar{\omega}_{E}^{1}=\left(\left[\left(z_{2}, 0\right),\left(z_{3}, 0\right)\right],\left[\left(z_{5}, 0\right),\left(z_{6}, 0\right)\right],\left[\left(z_{3}, 0\right),\left(z_{4}, 0\right)\right]\right)
$$




$$
\begin{aligned}
& l \bar{\omega}_{E}^{2}=\left(\left[\left(z_{6}, 0\right),\left(z_{7}, 0\right)\right],\left[\left(z_{1}, 0\right),\left(z_{3}, 0\right)\right],\left[\left(z_{4}, 0\right),\left(z_{5}, 0\right)\right]\right) \\
& l \bar{\omega}_{E}^{3}=\left(\left[\left(z_{2}, 0\right),\left(z_{4}, 0\right)\right],\left[\left(z_{2}, 0\right),\left(z_{4}, 0\right)\right],\left[\left(z_{2}, 0\right),\left(z_{3}, 0\right)\right]\right) \\
& l \bar{\omega}_{E}^{4}=\left(\left[\left(z_{5}, 0\right),\left(z_{6}, 0\right)\right],\left[\left(z_{4}, 0\right),\left(z_{5}, 0\right)\right],\left[\left(z_{3}, 0\right),\left(z_{5}, 0\right)\right]\right)
\end{aligned}
$$

and transform the interval linguistic weight vector on the ordered set $Q$ into the 2-tuple linguistic weight vector

$$
\begin{gathered}
l \bar{w}_{Q}=\left(\left[\left(z_{2}, 0\right),\left(z_{3}, 0\right)\right],\left[\left(z_{3}, 0\right),\left(z_{4}, 0\right)\right],\right. \\
\left.\left[\left(z_{5}, 0\right),\left(z_{6}, 0\right)\right]\right) .
\end{gathered}
$$

Step 2: Form the model (8), we get the following model for the optimal linguistic weight vector on the expert set $E$ for the attribute $c_{1}$.

$$
\text { s.t. }\left\{\begin{array}{l}
15 \Delta^{-1}\left(z_{e_{1}}^{1}, \alpha_{e_{1}}^{1}\right)+15 \Delta^{-1}\left(z_{e_{2}}^{1}, \alpha_{e_{2}}^{1}\right)+ \\
14 \Delta^{-1}\left(z_{e_{3}}^{1}, \alpha_{e_{3}}^{1}\right)-\lambda \sum_{k=1}^{3} \Delta^{-1}\left(z_{e_{k}}^{1}, \alpha_{e_{k}}^{1}\right)=0 \\
\Delta^{-1}\left(z_{e_{1}}^{1}, \alpha_{e_{1}}^{1}\right) \in[2,3], \Delta^{-1}\left(z_{e_{2}}^{1}, \alpha_{e_{2}}^{1}\right) \in[5,6], \\
\Delta^{-1}\left(z_{e_{3}}^{1}, \alpha_{e_{3}}^{1}\right) \in[3,4]
\end{array} .\right.
$$

Solve the above model, it has $\Delta^{-1}\left(z_{e_{1}}^{1}, \alpha_{e_{1}}^{1}\right)=2$, $\Delta^{-1}\left(z_{e_{2}}^{1}, \alpha_{e_{2}}^{1}\right)=5$, and $\Delta^{-1}\left(z_{e_{3}}^{1}, \alpha_{e_{3}}^{1}\right)=4$.

Similar to the calculation of the optimal linguistic weight vector on the expert set $E$ for the attribute $c_{1}$, we get the following optimal linguistic weight vectors on the expert set $E$ for the attributes $c_{j}$ $(j=2,3,4)$.

$$
\begin{aligned}
& \Delta^{-1}\left(z_{e_{1}}^{2}, \alpha_{e_{1}}^{2}\right)=6, \Delta^{-1}\left(z_{e_{2}}^{2}, \alpha_{e_{2}}^{2}\right)=1, \Delta^{-1}\left(z_{e_{3}}^{2}, \alpha_{e_{3}}^{2}\right)=4 ; \\
& \Delta^{-1}\left(z_{e_{1}}^{3}, \alpha_{e_{1}}^{3}\right)=2, \Delta^{-1}\left(z_{e_{2}}^{3}, \alpha_{e_{2}}^{3}\right)=2, \Delta^{-1}\left(z_{e_{3}}^{3}, \alpha_{e_{3}}^{3}\right)=2 ; \\
& \Delta^{-1}\left(z_{e_{1}}^{4}, \alpha_{e_{1}}^{4}\right)=6, \Delta^{-1}\left(z_{e_{2}}^{4}, \alpha_{e_{2}}^{4}\right)=4, \Delta^{-1}\left(z_{e_{3}}^{4}, \alpha_{e_{3}}^{4}\right)=3 .
\end{aligned}
$$

Step 3: Form the model (10), we get the following model for the optimal linguistic weight vector on the ordered set $Q$ for $i=j=1$.

$$
\min \lambda
$$

$$
\text { s.t. }\left\{\begin{array}{c}
\Delta^{-1}\left(z_{1}^{11}, \alpha_{1}^{11}\right)+\Delta^{-1}\left(z_{2}^{11}, \alpha_{2}^{11}\right)-\lambda \sum_{k=1}^{3} \Delta^{-1}\left(z_{k}^{11}, \alpha_{k}^{11}\right)=0 \\
\Delta^{-1}\left(z_{1}^{11}, \alpha_{1}^{11}\right)=\Delta^{-1}\left(z_{2}^{11}, \alpha_{2}^{11}\right) \in[2.5,3.5], \Delta^{-1}\left(z_{3}^{11}, \alpha_{3}^{11}\right) \in[5,6]
\end{array}\right. \text {. }
$$

Solve the above model, it has $\Delta^{-1}\left(z_{1}^{11}, \alpha_{1}^{11}\right)=\Delta^{-1}\left(z_{2}^{11}, \alpha_{2}^{11}\right)$ $=2.5$ and $\Delta^{-1}\left(z_{3}^{11}, \alpha_{3}^{11}\right)=5$.

Similar to the calculating of the optimal linguistic weight vector on the ordered set $Q$ for $i=j=1$, we get the following optimal linguistic weight matrix on the ordered set $Q$ for each pair $(i, j)(i=1,2,3,4,5 ; j=1,2,3$, 4).

$$
\begin{gathered}
L W_{Q}=\left(\left(\Delta^{-1}\left(z_{1}^{i j}, \alpha_{1}^{i j}\right), \Delta^{-1}\left(z_{2}^{i j}, \alpha_{2}^{i j}\right), \Delta^{-1}\left(z_{3}^{i j}, \alpha_{3}^{i j}\right)\right)\right)_{5 \times 4} \\
=\left(\begin{array}{cccc}
(2.5,2.5,5) & (2.5,2.5,5) & (2.5,2.5,5) & (2,3,5) \\
(2.5,2.5,5) & (2,3,5) & (2.5,2.5,5) & (2,5,5) \\
(2.5,2.5,5) & (2,3,5) & (2,5,5) & (2,3,5) \\
(2.5,2.5,5) & (2,3,5) & (2,3,5) & (2,3,5) \\
(2,3,5) & (2,4,4) & (2,5,5) & (2,3,5)
\end{array}\right) .
\end{gathered}
$$

Step 4: Let $u_{k}=d_{i j}^{(k)}(k=1,2,3)$ for each pair $(i, j)(i=1$, $2,3,4,5 ; j=1,2,3,4)$. Use the IET-LHAW operator, we get the comprehensive 2-tuple linguistic value $\left(q_{i j}, \alpha_{i j}\right)$, e.g., $i=j=1$,

$$
\begin{aligned}
\left(q_{11}, \alpha_{11}\right)= & \text { IET-LHAW }{ }_{l w_{Q}^{11}, l \omega_{E}^{1}}\left(<u_{1},\left(q_{11}^{1}, 0\right)>,<u_{2},\left(q_{11}^{2}, 0\right)>,\right. \\
& \left.<u_{3},\left(q_{11}^{3}, 0\right)>\right) \\
= & \left(s_{4},-0.08\right) .
\end{aligned}
$$

Similar to the calculation of $\left(q_{11}, \alpha_{11}\right)$, we get the following comprehensive 2-tuple linguistic matrix

$$
\tilde{Q}=\left(\begin{array}{cccc}
\left(s_{4},-0.08\right) & \left(s_{4}, 0.17\right) & \left(s_{3},-0.5\right) & \left(s_{3}, 0.13\right) \\
\left(s_{2}, 0.31\right) & \left(s_{3},-0.49\right) & \left(s_{4}, 0\right) & \left(s_{4}, 0.49\right) \\
\left(s_{5},-0.08\right) & \left(s_{4},-0.32\right) & \left(s_{5},-0.33\right) & \left(s_{3},-0.1\right) \\
\left(s_{6}, 0.08\right) & \left(s_{4},-0.49\right) & \left(s_{4},-0.5\right) & \left(s_{4}, 0.14\right) \\
\left(s_{5},-0.31\right) & \left(s_{2},-0.37\right) & \left(s_{4},-0.33\right) & \left(s_{4},-0.15\right)
\end{array}\right) .
$$

Step 5: Transform the interval linguistic weight vector on the attribute set $C$ into the 2-tuple linguistic weight vector

$$
\begin{aligned}
l \bar{\omega}_{C}= & \left(\left[\left(z_{5}, 0\right),\left(z_{6}, 0\right)\right],\left[\left(z_{4}, 0\right),\left(z_{6}, 0\right)\right],\right. \\
& {\left.\left[\left(z_{3}, 0\right),\left(z_{4}, 0\right)\right],\left[\left(z_{5}, 0\right),\left(z_{7}, 0\right)\right]\right) }
\end{aligned}
$$

and the interval linguistic weight vector on the ordered set $N$ for each $i \quad(i=1,2,3,4,5)$ into the following 2-tuple linguistic weight vector

$$
\begin{aligned}
l \bar{w}_{N}^{i}= & \left(\left[\left(z_{1}, 0\right),\left(z_{2}, 0\right)\right],\left[\left(z_{2}, 0\right),\left(z_{3}, 0\right)\right],\right. \\
& {\left.\left[\left(z_{3}, 0\right),\left(z_{4}, 0\right)\right],\left[\left(z_{5}, 0\right),\left(z_{6}, 0\right)\right]\right) . }
\end{aligned}
$$

Step 6: From $\tilde{Q}=\left(\left(q_{i j}, \alpha_{i j}\right)\right)_{5 \times 4}$, it has

$$
\begin{aligned}
& \qquad \tilde{R}^{+}=\left(\left(s_{6}, 0.08\right),\left(s_{4}, 0.17\right),\left(s_{5},-0.33\right),\left(s_{4}, 0.49\right)\right) \\
& \text { and } \\
& \qquad \tilde{R}^{-}=\left(\left(s_{2}, 0.31\right),\left(s_{2},-0.37\right),\left(s_{3},-0.5\right),\left(s_{3},-0.1\right)\right) .
\end{aligned}
$$

From $\tilde{R}^{+}$and $\tilde{R}^{-}$, we get the distance matrices $D^{+}=\left(d_{i j}^{+}\right)_{5 \times 4}$ and $D^{-}=\left(d_{i j}^{-}\right)_{5 \times 4}$ as follows: 


$$
\begin{aligned}
D^{+} & =\left(\begin{array}{cccc}
2.16 & 0 & 2.17 & 1.36 \\
3.77 & 1.66 & 0.67 & 0 \\
1.16 & 0.49 & 0 & 1.59 \\
0 & 0.66 & 1.17 & 0.35 \\
1.39 & 2.54 & 1 & 0.64
\end{array}\right), \\
D^{-} & =\left(\begin{array}{cccc}
1.61 & 2.54 & 0 & 0.23 \\
0 & 0.88 & 1.5 & 1.59 \\
2.61 & 2.05 & 2.17 & 0 \\
3.77 & 1.88 & 1 & 1.24 \\
2.38 & 0 & 1.17 & 0.95
\end{array}\right) .
\end{aligned}
$$

From the model (12), we get the following model for the optimal linguistic weight vector on the attribute set $C$.

$\min \lambda$

$$
\text { s.t. }\left\{\begin{array}{l}
2.25 \Delta^{-1}\left(z_{c_{1}}, \alpha_{c_{1}}\right)+2.11 \Delta^{-1}\left(z_{c_{2}}, \alpha_{c_{2}}\right)+2.31 \Delta^{-1}\left(z_{c_{3}}, \alpha_{c_{3}}\right) \\
+2.48 \Delta^{-1}\left(z_{c_{4}}, \alpha_{c_{4}}\right)-\lambda \sum_{j=1}^{4} \Delta^{-1}\left(z_{c_{j}}, \alpha_{c_{j}}\right)=0 \\
\Delta^{-1}\left(z_{c_{1}}, \alpha_{c_{1}}\right) \in[5,6], \Delta^{-1}\left(z_{c_{2}}, \alpha_{c_{2}}\right) \in[4,6], \\
\Delta^{-1}\left(z_{c_{3}}, \alpha_{c_{3}}\right) \in[3,4], \Delta^{-1}\left(z_{c_{4}}, \alpha_{c_{4}}\right) \in[5,7]
\end{array} .\right.
$$

Solve the above model, it has

$$
\begin{aligned}
& \Delta^{-1}\left(z_{c_{1}}, \alpha_{c_{1}}\right)=5, \Delta^{-1}\left(z_{c_{2}}, \alpha_{c_{2}}\right)=4, \\
& \Delta^{-1}\left(z_{c_{3}}, \alpha_{c_{3}}\right)=3, \Delta^{-1}\left(z_{c_{4}}, \alpha_{c_{4}}\right)=5 .
\end{aligned}
$$

Step 7: From the model (14), we get the following model for the optimal linguistic weight vector on the ordered set $N$ for $i=1$.

$$
\text { s.t. }\left\{\begin{array}{l}
0.93 \Delta^{-1}\left(z_{1}^{1}, \alpha_{1}^{1}\right)+0.74 \Delta^{-1}\left(z_{2}^{1}, \alpha_{2}^{1}\right)+0.49 \Delta^{-1}\left(z_{3}^{1}, \alpha_{3}^{1}\right) \\
+0.3 \Delta^{-1}\left(z_{4}^{1}, \alpha_{4}^{1}\right)-\lambda \sum_{j=1}^{4} \Delta^{-1}\left(z_{j}^{1}, \alpha_{j}^{1}\right)=0 \\
\Delta^{-1}\left(z_{1}^{1}, \alpha_{1}^{1}\right) \in[1,2], \Delta^{-1}\left(z_{2}^{1}, \alpha_{2}^{1}\right) \in[2,3], \\
\Delta^{-1}\left(z_{3}^{1}, \alpha_{3}^{1}\right) \in[3,4], \Delta^{-1}\left(z_{4}^{1}, \alpha_{4}^{1}\right) \in[5,6]
\end{array} .\right.
$$

Solve the above model, it has

$$
\begin{aligned}
& \Delta^{-1}\left(z_{1}^{1}, \alpha_{1}^{1}\right)=1, \Delta^{-1}\left(z_{2}^{1}, \alpha_{2}^{1}\right)=2, \\
& \Delta^{-1}\left(z_{3}^{1}, \alpha_{3}^{1}\right)=3, \Delta^{-1}\left(z_{4}^{1}, \alpha_{4}^{1}\right)=6 .
\end{aligned}
$$

Similar to the calculating of the optimal linguistic weight vector on the ordered set $N$ for $i=1$, we get the following optimal linguistic weight vectors on the ordered set $N$ for $i=2,3,4,5$.

$\Delta^{-1}\left(z_{1}^{2}, \alpha_{1}^{2}\right)=1, \Delta^{-1}\left(z_{2}^{2}, \alpha_{2}^{2}\right)=2, \Delta^{-1}\left(z_{3}^{2}, \alpha_{3}^{2}\right)=3, \Delta^{-1}\left(z_{4}^{2}, \alpha_{4}^{2}\right)=5$, $\Delta^{-1}\left(z_{1}^{3}, \alpha_{1}^{3}\right)=1, \Delta^{-1}\left(z_{2}^{3}, \alpha_{2}^{3}\right)=2, \Delta^{-1}\left(z_{3}^{3}, \alpha_{3}^{3}\right)=3, \Delta^{-1}\left(z_{4}^{3}, \alpha_{4}^{3}\right)=6$, $\Delta^{-1}\left(z_{1}^{4}, \alpha_{1}^{4}\right)=1, \Delta^{-1}\left(z_{2}^{4}, \alpha_{2}^{4}\right)=2, \Delta^{-1}\left(z_{3}^{4}, \alpha_{3}^{4}\right)=3, \Delta^{-1}\left(z_{4}^{4}, \alpha_{4}^{4}\right)=6$,
$\Delta^{-1}\left(z_{1}^{5}, \alpha_{1}^{5}\right)=1, \Delta^{-1}\left(z_{2}^{5}, \alpha_{2}^{5}\right)=2, \Delta^{-1}\left(z_{3}^{5}, \alpha_{3}^{5}\right)=4, \Delta^{-1}\left(z_{4}^{5}, \alpha_{4}^{5}\right)=6$. Step 8: Let $u_{j}=d_{i j}(j=1,2,3,4)$ for each $i(i=1,2,3,4,5)$. By the IET-LHAW operator, we obtain the comprehensive 2-tuple linguistic value $\left(q_{i}, \alpha_{i}\right)$ of alternative $a_{i}$, e.g. $i=1$,

$$
\begin{aligned}
\left(q_{1}, \alpha_{1}\right)= & \text { IET-LHAW }_{l w_{N}^{1}, l \omega_{C}}\left(<u_{1},\left(q_{11}, \alpha_{11}\right)>,<u_{2},\left(q_{12}, \alpha_{12}\right)>,\right. \\
& \left.<u_{3},\left(q_{13}, \alpha_{13}\right)>,<u_{4},\left(q_{14}, \alpha_{14}\right)>\right) \\
= & \left(s_{3}, 0.45\right) .
\end{aligned}
$$

Similar to the calculation of $\left(q_{1}, \alpha_{1}\right)$, we get the comprehensive 2-tuple linguistic values $\left(q_{i}, \alpha_{i}\right)$ of alternatives $a_{i}(i=2,3,4,5)$ as follows:

$$
\begin{gathered}
\left(q_{2}, \alpha_{2}\right)=\left(s_{3}, 0.23\right),\left(q_{3}, \alpha_{3}\right)=\left(s_{4}, 0.04\right), \\
\left(q_{4}, \alpha_{4}\right)=\left(s_{4}, 0.11\right),\left(q_{5}, \alpha_{5}\right)=\left(s_{3}, 0.8\right) .
\end{gathered}
$$

Step 9: According to the comprehensive 2-tuple linguistic values $\left(q_{i}, \alpha_{i}\right) \quad(i=1,2,3,4,5)$ and the relationship between 2-tuple linguistic arguments [5], it has

$$
\left(q_{2}, \alpha_{2}\right)<\left(q_{1}, \alpha_{1}\right)<\left(q_{5}, \alpha_{5}\right)<\left(q_{3}, \alpha_{3}\right)<\left(q_{4}, \alpha_{4}\right) .
$$

Namely, $a_{4}$ (arms company) is the best choice.

In the following, we use the IET-LHGM operator to get the best choice(s) in the above example.

Step1'-Step 3': See Step1-Step 3.

Step 4': Let $u_{k}=d_{i j}^{(k)}$ ' $(k=1,2,3)$ for each pair $(i, j)$ $(i=1,2,3,4,5 ; j=1,2,3,4)$. Use the IET-LHGM operator, we obtain the comprehensive 2-tuple linguistic value $\left(q_{i j}^{\prime}, \alpha_{i j}^{\prime}\right)$, e.g. $i=j=1$,

$$
\begin{aligned}
\left(q_{11}^{\prime}, \alpha_{11}^{\prime}\right)= & \text { IET-LHGW }{ }_{l w_{Q}^{\prime 1}, l \omega_{E}^{\prime}}\left(<u_{1},\left(q_{11}^{1}, 0\right)>,<u_{2},\left(q_{11}^{2}, 0\right)>,\right. \\
& \left.<u_{3},\left(q_{11}^{3}, 0\right)>\right) \\
= & \left(s_{4},-0.16\right) .
\end{aligned}
$$

Similar to the calculation of $\left(q_{i j}^{\prime}, \alpha_{i j}^{\prime}\right)$, we get the following comprehensive 2-tuple linguistic matrix

$$
\tilde{Q}^{\prime}=\left(\begin{array}{cccc}
\left(s_{4},-0.16\right) & \left(s_{4}, 0.06\right) & \left(s_{2}, 0.45\right) & \left(s_{3},-0.04\right) \\
\left(s_{2}, 0.27\right) & \left(s_{2},-0.27\right) & \left(s_{4},-0.06\right) & \left(s_{4}, 0.39\right) \\
\left(s_{5},-0.15\right) & \left(s_{4},-0.45\right) & \left(s_{5},-0.41\right) & \left(s_{2}, 0.19\right) \\
\left(s_{6}, 0.02\right) & \left(s_{3}, 0.33\right) & \left(s_{3}, 0.15\right) & \left(s_{4},-0.01\right) \\
\left(s_{4},-0.21\right) & \left(s_{2},-0.46\right) & \left(s_{4},-0.44\right) & \left(s_{3}, 0.24\right)
\end{array}\right) .
$$

Step 5': See Step 5.

Step 6': By the model (12), we get the following model for the optimal linguistic weight vector on the attribute set $C$.

$\min \lambda$ 


$$
\text { s.t. }\left\{\begin{array}{l}
2.49 \Delta^{-1}\left(z_{c_{1}}, \alpha_{c_{1}}\right)+2.2 \Delta^{-1}\left(z_{c_{2}}, \alpha_{c_{2}}\right)+2.46 \Delta^{-1}\left(z_{c_{3}}, \alpha_{c_{3}}\right) \\
+2.35 \Delta^{-1}\left(z_{c_{4}}, \alpha_{c_{4}}\right)-\lambda \sum_{j=1}^{4} \Delta^{-1}\left(z_{c_{j}}, \alpha_{c_{j}}\right)=0 \\
\Delta^{-1}\left(z_{c_{1}}, \alpha_{c_{1}}\right) \in[5,6], \Delta^{-1}\left(z_{c_{2}}, \alpha_{c_{2}}\right) \in[4,6], \\
\Delta^{-1}\left(z_{c_{3}}, \alpha_{c_{3}}\right) \in[3,4], \Delta^{-1}\left(z_{c_{4}}, \alpha_{c_{4}}\right) \in[5,7]
\end{array} .\right.
$$

Solve the above model, it has

$$
\begin{aligned}
& \Delta^{-1}\left(z_{c_{1}}, \alpha_{c_{1}}\right)=5, \Delta^{-1}\left(z_{c_{2}}, \alpha_{c_{2}}\right)=4, \\
& \Delta^{-1}\left(z_{c_{3}}, \alpha_{c_{3}}\right)=3, \Delta^{-1}\left(z_{c_{4}}, \alpha_{c_{4}}\right)=5 .
\end{aligned}
$$

Step 7': From the model (14), we get the following model for the optimal linguistic weight vector on the ordered set $N$ for $i=1$.

$$
\text { s.t. }\left\{\begin{array}{l}
0.88 \Delta^{-1}\left(z_{1}^{1}, \alpha_{1}^{1}\right)+0.73 \Delta^{-1}\left(z_{2}^{1}, \alpha_{2}^{1}\right)+0.51 \Delta^{-1}\left(z_{3}^{1}, \alpha_{3}^{1}\right) \\
+0.37 \Delta^{-1}\left(z_{4}^{1}, \alpha_{4}^{1}\right)-\lambda \sum_{j=1}^{4} \Delta^{-1}\left(z_{j}^{1}, \alpha_{j}^{1}\right)=0 \\
\Delta^{-1}\left(z_{1}^{1}, \alpha_{1}^{1}\right) \in[1,2], \Delta^{-1}\left(z_{2}^{1}, \alpha_{2}^{1}\right) \in[2,3], \\
\Delta^{-1}\left(z_{3}^{1}, \alpha_{3}^{1}\right) \in[3,4], \Delta^{-1}\left(z_{4}^{1}, \alpha_{4}^{1}\right) \in[5,6]
\end{array} .\right.
$$

Solve the above model, it has

$$
\begin{aligned}
& \Delta^{-1}\left(z_{1}^{1}, \alpha_{1}^{1}\right)=1, \Delta^{-1}\left(z_{2}^{1}, \alpha_{2}^{1}\right)=2, \\
& \Delta^{-1}\left(z_{3}^{1}, \alpha_{3}^{1}\right)=3, \Delta^{-1}\left(z_{4}^{1}, \alpha_{4}^{1}\right)=6 .
\end{aligned}
$$

Similar to the calculation of the optimal linguistic weight vector on the ordered set $N$ for $i=1$, we get the following optimal linguistic weight vectors on the ordered set $N$ for $i=2,3,4,5$.

$\Delta^{-1}\left(z_{1}^{2}, \alpha_{1}^{2}\right)=1, \Delta^{-1}\left(z_{2}^{2}, \alpha_{2}^{2}\right)=2.5, \Delta^{-1}\left(z_{3}^{2}, \alpha_{3}^{2}\right)=2.5, \Delta^{-1}\left(z_{4}^{2}, \alpha_{4}^{2}\right)=5$, $\Delta^{-1}\left(z_{1}^{3}, \alpha_{1}^{3}\right)=1, \Delta^{-1}\left(z_{2}^{3}, \alpha_{2}^{3}\right)=2, \Delta^{-1}\left(z_{3}^{3}, \alpha_{3}^{3}\right)=3, \Delta^{-1}\left(z_{4}^{3}, \alpha_{4}^{3}\right)=6$, $\Delta^{-1}\left(z_{1}^{4}, \alpha_{1}^{4}\right)=1, \Delta^{-1}\left(z_{2}^{4}, \alpha_{2}^{4}\right)=2, \Delta^{-1}\left(z_{3}^{4}, \alpha_{3}^{4}\right)=3, \Delta^{-1}\left(z_{4}^{4}, \alpha_{4}^{4}\right)=6$, $\Delta^{-1}\left(z_{1}^{5}, \alpha_{1}^{5}\right)=1, \Delta^{-1}\left(z_{2}^{5}, \alpha_{2}^{5}\right)=2, \Delta^{-1}\left(z_{3}^{5}, \alpha_{3}^{5}\right)=3, \Delta^{-1}\left(z_{4}^{5}, \alpha_{4}^{5}\right)=6$. Step 8': Let $u_{j}=d^{\prime}{ }^{\prime}{ }_{i j}(j=1,2,3,4)$ for each $i$ $(i=1,2,3,4,5)$. By the IET-LHGM operator, we obtain the comprehensive 2-tuple linguistic value $\left(q_{i}^{\prime}, \alpha_{i}^{\prime}\right)$ of alternative $a_{i}$, e.g. $i=1$,

$$
\begin{aligned}
& \left(q_{1}^{\prime}, \alpha_{1}^{\prime}\right)=\operatorname{IET}-\mathrm{LHGW}_{l w_{N}^{\prime}, \omega_{C}}\left(\left\langleu_{1},\left(q_{11}^{\prime}, \alpha_{11}^{\prime}\right)>,\left\langle u_{2},\left(q_{12}^{\prime}, \alpha_{12}^{\prime}\right)>\right.\text {, }\right.\right. \\
& \left.<u_{3},\left(q_{13}^{\prime}, \alpha_{13}^{\prime}\right)>,<u_{4},\left(q_{14}^{\prime}, \alpha_{14}^{\prime}\right)>\right) \\
& =\left(s_{3}, 0.29\right) \text {. }
\end{aligned}
$$

Similar to the calculation of $\left(q_{1}^{\prime}, \alpha_{1}^{\prime}\right)$, we get the comprehensive 2-tuple linguistic values $\left(q_{i}^{\prime}, \alpha_{i}^{\prime}\right)$ of alternatives $a_{i}(i=2,3,4,5)$ as follows:

$$
\begin{aligned}
& \left(q_{2}^{\prime}, \alpha_{2}^{\prime}\right)=\left(s_{3},-0.02\right),\left(q_{3}^{\prime}, \alpha_{3}^{\prime}\right)=\left(s_{4},-0.22\right), \\
& \left(q_{4}^{\prime}, \alpha_{4}^{\prime}\right)=\left(s_{4},-0.12\right),\left(q_{5}^{\prime}, \alpha_{5}^{\prime}\right)=\left(s_{3}, 0.21\right) .
\end{aligned}
$$

Step 9': According to the comprehensive 2-tuple linguistic values $\left(q_{i}^{\prime}, \alpha_{i}^{\prime}\right)(i=1,2,3,4,5)$ and the relationship between 2-tuple linguistic arguments [5], it gets

$$
\left(q_{2}^{\prime}, \alpha_{2}^{\prime}\right)<\left(q_{5}^{\prime}, \alpha_{5}^{\prime}\right)<\left(q_{1}^{\prime}, \alpha_{1}^{\prime}\right)<\left(q_{3}^{\prime}, \alpha_{3}^{\prime}\right)<\left(q_{4}^{\prime}, \alpha_{4}^{\prime}\right) \text {. }
$$

Namely, $a_{4}$ (arms company) is the best choice.

In this example, by the IET-LHAW and IET-LHGM operators we obtain the slightly different ranking results, but the best choice is the alternative $a_{4}$ in both the cases.

\section{Conclusions}

From the given example in Section 2, we know that if the HWA or HWGM operator is directly applied to aggregate a set of linguistic variables for the predefined linguistic term set $S$, then it cannot guarantee the aggregation value fall in the scope of $S$. As we know, the same number's arithmetic or geometric average equals to itself. However, for the HWA or HWGM operator this conclusion does not hold, which contradicts people's intuition.

We have defined four aggregation operators for 2-tuple linguistic variables. These operators do not only consider the importance of the elements but also reflect the importance of their ordered positions. It is worth pointing out that most of the existing linguistic aggregation operators are special cases of our operators. When the information about linguistic weight vectors is partly known, the models for the optimal linguistic weight vectors on the expert set, on the attribute set and on their ordered sets are built, respectively. An approach to multi-attribute group decision making under linguistic environment is developed, where the weights of experts, attributes and their ordered positions all take the form of linguistic arguments. Finally, a financial decisionmaking problem under linguistic environment has been given to illustrate our approach.

\section{Acknowledgement}

This work was supported by the National Natural Science Foundation Youth Project of China (No. 71201089), the National Natural Science Foundation of China (Nos. 71071018 and 71271217), the Natural Science Foundation Youth Project of Shandong Province, China (ZR2012GQ005), and the Specialized Research Fund for the Doctoral Program of Higher Education (No. 20111101110036).

\section{References}

[1] F. Herrera, E. Herrera-Viedma and J. L. Verdegay, A 
linguistic decision process in group decision-making, Group Decis. Negot. 5(1) (1996) 165-176.

[2] I. Palomares, J. Liu, Y. Xu, and L. Martínez, Modelling experts' attitudes in group decision making, Soft Comput. 16(10) (2012) 1755-1766.

[3] L. Martínez, Sensory evaluation based on linguistic decision analysis, Int. J. Approx. Reason. 44(2) (2007) 148-164.

[4] F. Herrera and E. Herrera-Viedma, Linguistic decision analysis: steps for solving decision problems under linguistic information, Fuzzy Sets Syst. 115(1) (2000) 67-82.

[5] F. Herrera and L. Martínez, A 2-tuple fuzzy linguistic representation model for computing with words, IEEE Trans. Fuzzy Syst. 8(6) (2000) 746-752.

[6] F. Herrera, E. Herrera-Viedma and L. Martínez, A fusion approach for managing multi-granularity linguistic term sets in decision-making, Fuzzy Sets Syst. 114(1) (2000) 43-58.

[7] F. Herrera, E. López and M. A. Rodríguez, A linguistic decision model for promotion mix management solved with genetic algorithms, Fuzzy Sets Syst. 131(1) (2002) 47-61.

[8] L. Martínez and F. Herrera, An overview on the 2-tuple linguistic model for computing with words in decision making: Extensions, applications and challenges, Inform. Sci. 207(10) (2012) 1-18.

[9] G. W. Wei, Extension of TOPSIS method for 2-tuple linguistic multiple attribute group decision making with incomplete weight information. Knowl. Inf. Syst. 25(3) (2010) 623-634.

[10] Z. S. Xu, A note on linguistic hybrid arithmetic averaging operator in multiple attribute group decision making with linguistic information, Group Decis. Negot. 15(6) (2006) 593-604.

[11] Z. S. Xu, A method for multiple attribute decision making with incomplete weight information in linguistic setting, Knowl-Based Syst. 20(8) (2007) 719-725.

[12] Z. S. Xu, Extended IOWG operator and its use in group decision making based on multiplicative linguistic preference relations, Am. J. Appl. Sci. 2(3) (2005) 633-643.

[13] E. Herrera-Viedma and A. G. Lopez-Herrera, A model of information retrieval system with unbalanced fuzzy linguistic information, Int. J. Intel. Syst. 22(11) (2007) 1197-1214.

[14] E. Herrera-Viedma, A. G. Lopez-Herrera, M. Luque and C. Porcel, A fuzzy linguistic IRS model based on a 2-tuple fuzzy linguistic approach, Int. J. Uncertain. Fuzz. 15(2) (2007) 225-250.

[15] E. Herrera-Viedma and A. G. Lopez-Herrera, A review on information accessing systems based on fuzzy linguistic modeling, Int. J. Comput. Intel. Syst. 3(4) (2010) 420-437.

[16] N. Fenton and W. Wang, Risk and confidence analysis for fuzzy multicriteria decision making, Knowl-Based Syst. 19(6) (2006) 430-437.
[17] J. Liu, L. Martínez, H. Wang, R. M. Rodríquez and V. Novozhilov, Computing with words in risk assessment, Int. J. Comput. Intel. Syst. 3(4) (2010) 396-419.

[18] L. Martínez, J. Liu, D. Ruan and J. B. Yang, Dealing with heterogeneous information in engineering evaluation processes, Inform. Sci. 177(7) (2007) $1533-1542$.

[19] R. de Andrés, J. L. García-Lapresta and L. Martínez, A multi-granular linguistic model for management decision-making in performance appraisal, Soft Comput. 14(1) (2010) 21-34.

[20] J. L. García-Lapresta, B. Llamazares and M. Martínez- Panero, A social choice analysis of the Borda rule in a general linguistic framework, Int. J. Comput. Intel. Syst. 3(4) (2010) 501-513.

[21] H. Ishibuchi, T. Nakashima and M. Nii, Classification and modeling with linguistic information granules: advanced approaches to linguistic data mining, Springer, Berlin, 2004.

[22] Y. P. Jiang and Z. P. Fan, Property analysis of the aggregation operators for 2-tuple linguistic information, Control and Decision 18(6) (2003) 754-757. (in chinese)

[23] Y. Zhang and Z. P. Fan, An approach to linguistic multiple attribute decision making with linguistic information based on ELOWA operator, Systems Engineering 24(12) (2006) 98-101. (in chinese)

[24] F. Wei, C. A. Liu and S. Y. Liu, A method for group decision making with linguistic information based on uncertain information processing, Oper. Res. Manage. Sci. 15(3) (2006) 31-35. (in chinese)

[25] Y. J. Xu and L. Huang, An approach to group decision making problems based on 2-tuple linguistic aggregation operators, ISECS, Guangzhou, China, 2008, pp. 73-77.

[26] G. W. Wei, A method for multiple attribute group decision making based on the ET-WG and ET-OWG operators with 2-tuple linguistic information, Expert Syst. Appl., 37(12) (2010) 7895-7900.

[27] Y. J. Xu and H. M. Wang, Approaches based on 2-tuple linguistic power aggregation operators for multiple attribute group decision making under linguistic environment, Applied Soft Comput. 11(5) (2011) 3988-3997.

[28] G. W. Wei, Some generalized aggregating operators with linguistic information and their application to multiple attribute group decision making, Comput. Ind. Eng. 61(1) (2011) 32-38.

[29] Z. S. Xu and Q. L. Da, An overview of operators for aggregating information, Int. J. Intel. Syst. 18(9) (2003) 953-969.

[30] Z. S. Xu and Q. L. Da, An overview of operators for aggregating information, Int. J. Intel. Syst. 18(9) (2003) 953-969.

[31] Z. S. Xu and Da, Q.L. Combined weighted geometric averaging operator and its application, J. Southeast Univ. 32 (2002) 506-509. 
[32] Z. S. Xu, A method based on linguistic aggregation operators for group decision making with linguistic preference relations, Inform. Sci. 166(1) (2004) 19-30.

[33] Z. S. Xu, Uncertain linguistic aggregation operators based approach to multiple attribute group decision making under uncertain linguistic environment, Inform. Sci. 168(1-4) (2004) 171-184.

[34] G. W. Wei, Uncertain linguistic hybrid geometric mean operator and its Application to group decision making under uncertain linguistic environment, Int. $J$ Uncertain. Fuzz. 17(2) (2009) 251- 267.

[35] R. R. Yager, On ordered weighted averaging aggregation operators in multicriteria decision making, IEEE T. Syst. Man Cy. 18 (1988) 183-190.

[36] V. Torra, The weighted OWA operator, Int. J. Intel. Syst. 12(2) (1997) 153-166.

[37] Z. S. Xu and R. R. Yager, Some geometric aggregation operators based on intuitionistic fuzzy sets. Int. J. Gen. Syst. 35(4) (2006) 417-433.

[38] Z. S. Xu, An approach based on the uncertain LOWG and induced uncertain LOWG operators to group decision making with uncertain multiplicative linguistic preference relations, Decision Support Systems 41(2) (2006) 488-499.

[39] Z. S. Xu, Induced uncertain linguistic OWA operators applied to group decision making, Inform. Fusion 7(2) (2006) 231-238.

[40] C. L. Hwang and K, Yoon, Multiple attribute decision making methods and applications, Springer, Berlin Heidelberg, 1981.

[41] M. Delgado, F. Herrera, E. Herrera-Viedma and L. Martinez, Combining numerical and linguistic information in group decision making, Inform. Sci. 107(1-4) (1998) 177-194. 